\begin{tabular}{c|c|c|c|} 
SELECCIONES MATEMÁTICAS \\
Universidad Nacional de Trujillo \\
ISSN: $2411-1783$ (Online) \\
2021; Vol. 8(2): 295-315.
\end{tabular}

\title{
Mathematical analysis of a symptom-structured epidemic model
}

\section{Análisis matemático de un modelo epidémico estructurado por síntomas}

\author{
Pedro Isaac Pesantes-Grados ${ }^{\circledR}$ and Roxana López-Cruz ${ }^{\mathbb{1}}$
}

Received, Set. 30, 2021

Accepted, Dec. 10, 2021

How to cite this article:

Pesantes-Grados P, López-Cruz R. Mathematical analysis of a symptom-structured epidemic model. Selecciones Matemáticas. 2021;8(2):295-315. http://dx.doi.org/10.17268/sel .mat.2021.02.07

\begin{abstract}
We propose a modification of the SLIAR (Susceptible- Latent- Symptomatic Infected-Asymptomatic- Recovered) mathematical-epidemiological model with vital dynamics. This model includes a vaccination control strategy, and a treatment to reduce the symptoms, also the symptomatic infected population is divided into two states according to its severity. The qualitative analysis shows the local stability of the disease-free equilibrium point and the endemic point. Simulations and the statistical sensitivity analysis are developed using a set of parameters for COVID-19, and we found that the transmission, recruitment, finally, vaccination rates are potential targets to control an outbreak.
\end{abstract}

Keywords . Vaccination, stability, sensitivity, COVID-19.

\section{Resumen}

Se propone una modificación del modelo epidemiológico SLIAR (Susceptible-Latente-Infectado SintomáticoAsintomático-Recuperado) con dinámica vital. Este modelo incluye una estrategia de control por vacunación, y un tratamiento para reducir los síntomas, además la población infectada sintomática se divide en dos estados según su gravedad. El análisis cualitativo muestra la estabilidad local del punto de equilibrio libre de enfermedad y el punto endémico. Las simulaciones y el análisis estadístico de sensibilidad son desarrollados utilizando un conjunto de parámetros para COVID-19, finalmente, encontramos que las tasas de transmisión, reclutamiento y vacunación son objetivos potenciales para controlar un brote epidémico.

Palabras clave. Vacunación, estabilidad, sensibilidad, COVID-19.

1. Introduction. In the development of mathematical models applied to epidemiology, an important milestone is the SIR model proposed by Kermack and McKendrick [13], which classifies epidemiological populations into three states: Susceptible (S) as the population at risk of contracting the disease, infected (I) as those who possess the disease and are able to spread it and removed (R) which can be considered both recovered and deceased. This model has served as a basis for the development of numerous epidemiological models, which allow to capture in greater detail either a novel theoretical approach or an application to a particular disease.

Brauer [8] proposed an influenza SEI (susceptible-exposed-infected) mathematical model where considered an asymptomatic compartment in lieu of the exposed. Arino et al. [4] proposed a basic SLIAR model and one with treatment considering the following epidemiological states for a respiratory disease in general: Susceptible (S), latent (L) that are infected people who can not yet spread the disease, and among those infected that propagate the disease are considered symptomatic (I) and asymptomatic (A), finally it is considered the removed or recovered population (R). The SLIAR model incorporates asymptomatic status

\footnotetext{
*Facultad de Ciencias Matemáticas, Universidad Nacional Mayor de San Marcos, Av. Venezuela S/N, Lima 01, Lima, Perú. (pedro.pesantes@unmsm. edu.pe).

${ }^{\dagger}$ Facultad de Ciencias Matemáticas, Universidad Nacional Mayor de San Marcos, Av. Venezuela S/N, Lima 01, Lima, Perú. (rlopezc@unmsm. edu.pe).
} 
based on a characteristic of influenza, in which cases of infectious diseases that do not develop symptoms occur. It was developed with the aim of analyzing the dynamics of basic models in the post-pandemic context of avian influenza (H5N1) and SARS (2002-2003). This research concluded that the proposed models were sensitive to parameter values, in particular the fraction of dormant patients which develop symptoms.

In the context of COVID-19 pandemic (Coronavirus 19 disease, caused by the SARS-CoV-2 virus, which belongs to the family Coronaviridae), this disease triggers a myriad of pathophysiological disorders, whose most striking characteristic is its rapid transmissibility, it also presents a series of symptomatological characteristics that range from lack of symptoms (asymptomatic) to moderate pathological effects such as lung inflammation and thrombosis, generating in many cases severe acute respiratory syndrome (SARS), a cytokines storm and until death [10]. Moreover, the capability to transmit the disease from asymptomatic was found [22]. It is necessary, in this context of pandemic, to develop tools that allow us to estimate the possible scenarios for local outbreaks of this disease, and to give us general guidelines on how to prepare to cope with it. Here, mathematical and computational modeling plays a major role, as it allows us to simulate and experiment in silico various hypotheses that will be empirically contrasted, but would otherwise be difficult to experiment directly with the population or wait long enough until sufficiently robust and meaningful data can be analyzed.

Based on the previous model of Arino et al. [4] and subsequent modifications [5, 6], they proposed a modification of latent, infected and asymptomatic compartments, dividing each one in two stages according to their degree of infectivity, this model has been applied to the case study of the current COVID-19 pandemic. In this proposal, the incubation state without infecting capacity and the state of pre-symptomatic infectious latent was differentiated, where the latter one could move to a state of symptomatic or asymptomatic infectious. The transitions among incubation compartments, asymptomatic/symptomatic infected with a higher probability of survival or not, were estimated using an Erlang distribution, i.e., a gamma distribution function with form two parameter. For the estimation of parameters, data from various sources was used and algorithms encoded in R language were used for processing [7].

In the current situation, under the pandemic caused by the SARS-CoV-2 virus, the review and updating of some hypotheses like the existence of pharmacological treatment, vaccination schemes or different infected types takes important relevance, as well as studying the dynamics of mathematical models related to infectious-contagious respiratory diseases.

This work examines the dynamics and local stability of the SLIIAR (Susceptible-Latent-mild symptomatic Infected -severe symptomatic Infected -Asymptomatic-Recovered) model, which is derived from the SLIAR model and incorporates a new compartment to stratify the symptomatic infected people and vital dynamics. Besides, due to the characteristics of influenza, SARS, and COVID-19, we consider implementing the vaccination effect with total immunity and a parameter that represents the treatment effect. A qualitative analysis was done to establish the positivity, boundedness, existence of the equilibrium points, and their local stability. Additionally, we perform a sensitivity analysis in order to find the most relevant parameters that perturb the disease dynamics. These analyses help us to determine the relevance of some sanitary measures in an outbreak.

2. SLIAR model. This model has been previously analyzed using the AH2N2 influenza spread parameters of 1957, and has an advantage over SEIR models (Susceptible - Exposed - Infected - Removed), in the sense that asymptomatic can spread the disease [4].

\begin{tabular}{|c|c|c|c|}
\hline $\begin{array}{c}\text { Susceptible } \\
\text { stage }\end{array}$ & $\begin{array}{l}\text { Latent } \\
\text { period }\end{array}$ & Infectious period & $\begin{array}{c}\text { Removal stage } \\
\text { (Recovery, immunity } \\
\text { or death) }\end{array}$ \\
\hline & $\begin{array}{r}\text { Incuba } \\
\text { peric }\end{array}$ & $\begin{array}{l}\text { Symptoms } \\
\text { period }\end{array}$ & time \\
\hline
\end{tabular}

Figure 2.1: Epidemiological stages. Modified from [9]

2.1. Hypothesis. The mathematical model proposed by Arino et al. [4] does not consider vital dynamics and consists of five differential equations representing the five epidemiological states: population of susceptible $(S(t)=S)$, population of latent or exposed $(L(t)=L)$, population of symptomatic infectious 
$(I(t)=I)$, population of asymptomatic $(A(t))$ and population of recovered $(R(t)=R)$ in an instant $t$. It also considers the following hypothesis:

1. Each infected individual has the same probability of transmitting the disease, that is the same expected number of contacts, then the transmission rate $\boldsymbol{\beta}>\mathbf{0}$ is constant and obeys the law of mass action.

2. The latent population $(L)$ has no capacity to transmit the disease. Taking into account that the latency period is the time that elapses from infection until the person becomes infectious while the incubation period is the time that elapses until onset of symptoms [9], as it can see in the figure 2.1 .

3. In transmission dynamics, a fraction $p$ of the latent population transitions to the symptomatic infectious population compartment $(I)$ with a transition rate from infected to contagious $\kappa$; while, the remaining fraction of latents $(1-p)$ transits to the asymptomatic infectious compartment $(A)$ with the same transition rate $\kappa$.

4. The infection spreads through contact with members of the susceptible population and the infected population (symptomatic and asymptomatic); however, the asymptomatic $(A)$ infect with less intensity than the symptomatic $(I)$, with an infectivity rate reduced by a factor of $\delta$.

5. The removal takes place through a removal rate $\boldsymbol{\alpha}>\mathbf{0}$ for symptomatic cases, where a fraction of them $(f)$ are recovered and passed into the recovered compartment $(R)$, while the rest $(1-f)$ die from the infection. The removal rate $\boldsymbol{\eta}>\mathbf{0}$ for asymptomatic infectives transiting to the recovered compartment $(R)$.

Flow diagram of the model:

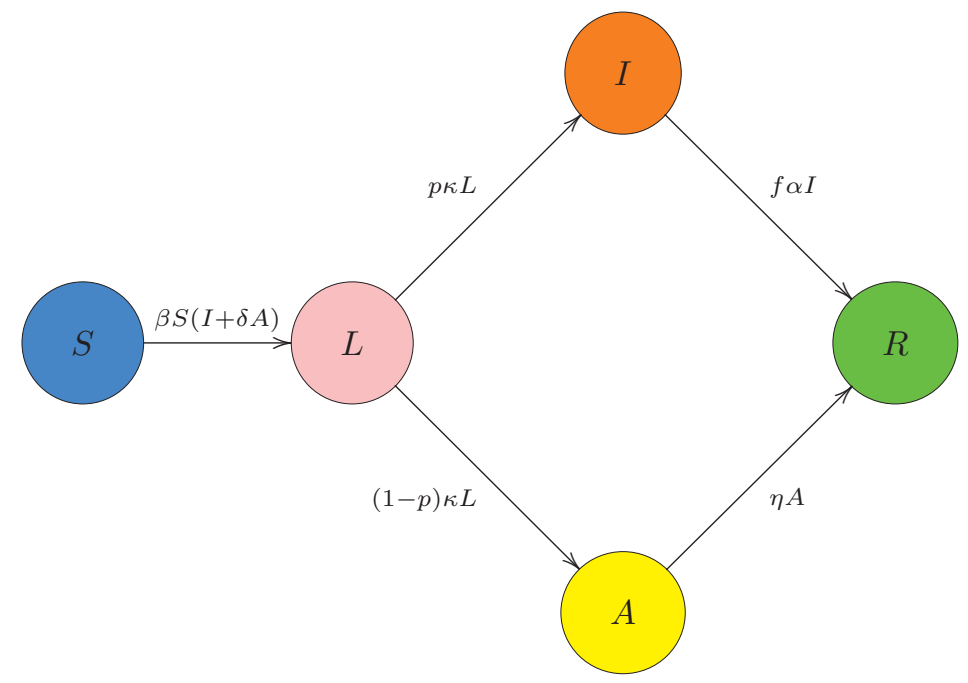

Figure 2.2: Flow diagram of SLIAR model [4]

Where:

- $\beta$, represents the constant transmission rate.

- $\delta$, is the rate of attenuation of infectivity by asymptomatics $(0 \leq \delta \leq 1)$.

- $\kappa$, is the rate of transition from latent to infected infectious.

- $\frac{1}{\kappa}$, in the number of days a dormant individual becomes infectious (symptomatic or asymptomatic).

- $p$, is the proportion of the latent population that becomes symptomatic infectious $(0 \leq p \leq 1)$.

- $(1-p)$, is the proportion of the latent population that becomes asymptomatic infectious.

- $\alpha$, is the rate of infection removal for symptomatic infected.

- $\frac{1}{\alpha}$, in the number of days that a symptomatic infectious individual becomes removed (recovers or dies).

- $\eta$, is the recovery rate of asymptomatics.

- $\frac{1}{\eta}$, in the number of days an asymptomatic infectious individual recovers.

- $f$, is the proportion of symptomatic infections that recover $(0 \leq f \leq 1)$.

- $(1-f)$, is the proportion of symptomatic infectious deceased.

While the flow diagram and the system representing them have not been considered the compartment of dead from disease, it is possible to add this, for a similar analysis. The ordinary differential equations system is the following: 


$$
\begin{cases}\frac{d S}{d t}=-\beta S(I+\delta A), & S_{(0)}=S_{0}>0 \\ \frac{d L}{d t}=\beta S(I+\delta A)-\kappa L & L_{(0)}=L_{0} \geq 0 \\ \frac{d I}{d t}=p \kappa L-\alpha I, & I_{(0)}=I_{0} \geq 0 \\ \frac{d A}{d t}=(1-p) \kappa L-\eta A, & A_{(0)}=A_{0} \geq 0 \\ \frac{d R}{d t}=f \alpha I+\eta A & R_{(0)}=R_{0} \geq 0 \\ & (\beta, \delta, \kappa, \alpha, p, f, \eta) \in \mathbb{R}_{+}^{7} .\end{cases}
$$

And the total size of the population at the moment $t$ is given by $N(t)=S(t)+L(t)+I(t)+A(t)+R(t)$.

2.2. Basic qualitative properties. This section will determine some of the properties for the mathematical analysis of the solutions for $t \geq 0$, such as the positivity and invariant region. For this purpose, the methodology outlined by Zhang et al. [29] for the demonstration of the lemmas will be followed.

2.2.1. Positivity. It is important to prove that all the variables of epidemiological states and the solutions of the system of differential equations (2.1) are not negative with positive initial conditions $\forall t \geq 0$.

Lemma 2.1. The system of differential equations (2.1) with initial conditions $S(0)>0, L(0) \geq$ $0, I(0) \geq 0, A(0) \geq 0, R(0) \geq 0$ has non negative solutions $S(t), L(t), I(t), A(t), R(t)$ for all $t \geq 0$.

Proof:

- Assume the solution $(S(t), L(t), I(t), A(t), R(t))$ of the system (2.1) with positive initial conditions of existence $(S(0)>0, L(0) \geq 0, I(0) \geq 0, A(0) \geq 0, R(0) \geq 0)$ in the interval $[0, b)$, where $0<b \leq \infty$. By the first equation of the system (2.1), we have to:

$$
S^{\prime}(t)=-\beta S(t)(I(t)+\delta A(t)),
$$

integrating we have to

$$
\begin{gathered}
\int_{0}^{t} S^{\prime}(t) d t=\int_{0}^{t}-\beta S(t)(I(t)+\delta A(t)) d t \\
\int_{0}^{t} \frac{S^{\prime}(t)}{S(t)} d t=\int_{0}^{t}-\beta(I(t)+\delta A(t)) d t \\
S(t)=S(0) e^{-\beta \int_{0}^{t}(I(\theta)+\delta A(\theta)) d \theta}>0, \forall t \in[0, b) .
\end{gathered}
$$

Therefore, there must be a $S(t)>0, \forall t \in[0, b)$.

- On the other hand, there will be a $t_{1} \in(0, b)$ such that $L\left(t_{1}\right)=0$ and $L(t)>0$ in the interval $\left[0, t_{1}\right)$. For any $t \in\left[0, t_{1}\right)$, by the third equation of the system (2.1):

$$
I^{\prime}(t)=p \kappa L(t)-\alpha I(t) \geq-\alpha I(t)
$$

integrating the differential inequality,

$$
\begin{gathered}
\int_{0}^{t} I^{\prime}(t) d t \geq \int_{0}^{t}-\alpha I(t) d t \\
\int_{0}^{t} \frac{I^{\prime}(t)}{I(t)} d t \geq-\alpha \int_{0}^{t} d t \\
I(t) \geq I(0) e^{-\alpha t}>0, \forall t \in\left(0, t_{1}\right) .
\end{gathered}
$$

- In addition, $\forall t \in\left[0, t_{1}\right]$, we have to

$$
L^{\prime}(t)=\beta S(t)(I(t)+\delta A(t))-\kappa L(t) \geq-\kappa L(t),
$$

integrating

$$
\int_{0}^{t_{1}} L^{\prime}(t) d t \geq \int_{0}^{t_{1}}-\kappa L(t) d t
$$




$$
\begin{gathered}
\int_{0}^{t_{1}} \frac{L^{\prime}(t)}{L(t)} d t \geq-\kappa \int_{0}^{t_{1}} d t \\
L\left(t_{1}\right) \geq L(0) e^{-\kappa t_{1}}>0, \forall t \in\left[0, t_{1}\right) .
\end{gathered}
$$

but this is a contradiction to $L\left(t_{1}\right)=0$, which was previously assumed. Therefore, $L(t)>0, \forall t \in$ $[0, b)$.

- Using the same methodology by contradiction, it can be shown that $I(t)>0, \forall t \in[0, b)$.

- For the fourth equation of the system (2.1), under the same methodology we have to

$$
A(t) \geq A(0) e^{-\eta t}>0, \forall t \in[0, b) .
$$

- For the fifth equation of the system (2.1), using the same methodology we obtain

$$
R^{\prime}(t)=f \alpha I(t)+\eta A(t),
$$

and integrating

$$
\begin{gathered}
\int_{0}^{t} R^{\prime}(t) d t=\int_{0}^{t}(f \alpha I(t)+\eta A(t)) d t \\
R(t)=\int_{0}^{t}(f \alpha I(\theta)+\eta A(\theta)) d \theta+R(0)>0, \forall t \in[0, b) .
\end{gathered}
$$

- We have thus obtained that

$$
S(t), L(t), I(t), A(t), R(t)>0, \forall t \in[0, b) .
$$

2.2.2. Invariant region. Because the model is related to biological populations, it is assumed that the variables and parameters are positive $\forall t \geq 0$. Therefore, the system of equations (2.1) within a region of the solutions space $\Omega$ of biological interest will be analyzed, which is presented in the following lemma.

Lemma 2.2. The feasible region $\Omega$, defined by

$$
\Omega=\left\{(S(t), L(t), I(t), A(t), R(t)) \in \mathbb{R}_{+}^{5}: 0 \leq N(t) \leq N(0)\right\},
$$

with initial conditions $S(0)>0, L(0) \geq 0, I(0) \geq 0, A(0) \geq 0, R(0) \geq 0$ is positively invariant to the system of differential equations (2.1).

Proof: Let the system of equations be (2.1), and adding them, we get

$$
\frac{d S}{d t}+\frac{d L}{d t}+\frac{d I}{d t}+\frac{d A}{d t}+\frac{d R}{d t}=-(1-f) \alpha I
$$

while $N(t)=S(t)+L(t)+I(t)+A(t)+R(t)$, we have to:

$$
\frac{d N}{d t}=-(1-f) \alpha I
$$

We integrate member to member in the interval of $t \in[0, t]$, we have to

$$
\begin{gathered}
\int_{0}^{t} N^{\prime}(\theta) d \theta=\int_{0}^{t}(-(1-f) \alpha I(\theta)) d \theta \\
N(t)=N(0)-(1-f) \alpha \int_{0}^{t} I(\theta) d \theta
\end{gathered}
$$

where it follows that

$$
N(t) \leq N(0) .
$$

where $N(0)$ represents the initial value of the total population. It proves the boundedness. In addition, using the previous result of positivity, we can conclude that $0 \leq N(t) \leq N(0)$. 


\subsection{Qualitative Analysis.}

2.3.1. Equilibrium points. In order to find the equilibrium solutions, we solve the system (2.1) by setting each equation equal to zero.

By simple inspection, we note that the trivial equilibrium point is a solution of the system, where $S=0, L=0, I=0, A=0, R=0$, however this point $E^{*}=(0,0,0,0,0)$ lacks biological interest in the context of an epidemic outbreak.

For $I=0$, we have a equilibrium point with $L=A=0$, where $R \geq 0$ and $S$ takes values from $0 \leq S \leq S_{0}$, then the disease-free equilibrium point is:

$$
E_{0}=\left(S_{0}, 0,0,0, R_{0}\right)
$$

2.3.2. Calculating $R_{0}$ using the next generation matrix. This methodology will be applied considering the steps followed by Amaya-Muvdi [3], where the subsystem of the infected compartments is evaluated directly:

$$
\left\{\begin{aligned}
\frac{d L}{d t} & =\beta S(I+\delta A)-\kappa L \\
\frac{d I}{d t} & =p \kappa L-\alpha I \\
\frac{d A}{d t} & =(1-p) \kappa L-\eta A .
\end{aligned}\right.
$$

From this subsystem, we define the following matrices:

- $\mathscr{F}_{(L, I, A)}$ : Matrix formed by the new infections appearance rates in the compartments of infected (matrix of entry of infected by the infection).

- $\mathscr{V}_{(L, I, A)}^{+}$: Matrix formed by the transfer rates of individuals to the infected compartments for reasons other than being infected (matrix of entry of infected by causes other than infection).

- $\mathscr{V}_{(L, I, A)}$ : Matrix formed by the transfer rates of individuals outside the infected compartments (infected exit matrix).

- $\mathscr{V}_{(L, I, A)}=\mathscr{V}_{(L, I, A)}^{-}-\mathscr{V}_{(L, I, A)}^{+}$.

According to the interpretation made by Amaya-Muvdi [3] and Diekmann et al. [11] of the methodology used, the matrix $\mathscr{F}_{(L, I, A)}+\mathscr{V}_{(L, I, A)}^{+}$evaluates the entries to the infected compartments, while the matrix $\mathscr{V}_{(L, I, A)}^{-}$represents the outputs of those compartments per unit of time. In addition, the components of the matrix $\mathscr{V}_{(L, I, A)}$ represent the expected time in which an individual who is in one of the epidemiological states $(L, I, A)$ passes to another of these states in the future. In our case:

$$
\begin{aligned}
& \mathscr{F}_{(L, I, A)}=\mathscr{F}=\left(\begin{array}{c}
\mathscr{F}_{L} \\
\mathscr{F}_{I} \\
\mathscr{F}_{A}
\end{array}\right)=\left(\begin{array}{c}
\beta S I+\beta \delta S A \\
0 \\
0
\end{array}\right) \\
& \mathscr{V}_{(L, I, A)}^{+}=\mathscr{V}^{+}=\left(\begin{array}{c}
\mathscr{V}_{L}^{+} \\
\mathscr{V}_{I}^{+} \\
\mathscr{V}_{A}^{+}
\end{array}\right)=\left(\begin{array}{c}
0 \\
p \kappa L \\
(1-p) \kappa L
\end{array}\right) \quad, \quad \mathscr{V}_{(L, I, A)}^{-}=\mathscr{V}^{-}=\left(\begin{array}{c}
\mathscr{V}_{L}^{-} \\
\mathscr{V}_{I}^{-} \\
\mathscr{V}_{A}^{-}
\end{array}\right)=\left(\begin{array}{c}
\kappa L \\
\alpha I \\
\eta A
\end{array}\right) \\
& \mathscr{V}_{(L, I, A)}=\mathscr{V}=\mathscr{V}_{(L, I, A)}^{-}-\mathscr{V}_{(L, I, A)}^{+}=\mathscr{V}^{-}-\mathscr{V}^{+}=\left(\begin{array}{c}
\kappa L \\
\alpha I-p \kappa L \\
\eta A-(1-p) \kappa L
\end{array}\right)
\end{aligned}
$$

We proceed to calculate the Jacobian or matrix derivatives of matrices $\mathscr{V}, \mathscr{F}$, evaluate them at the diseasefree equilibrium point for our case $E_{0}=\left(S_{0}, 0,0,0, R_{0}\right)$ :

$$
F=\left(\left.\frac{\partial \mathscr{F}_{i}}{\partial x_{i}}\right|_{\left(E_{0}\right)}\right) \quad, \quad V=\left(\left.\frac{\partial \mathscr{V}_{i}}{\partial x_{i}}\right|_{\left(E_{0}\right)}\right), \quad 1 \leq i, j \leq m
$$


Where: $\mathscr{F}_{i}, \mathscr{V}_{i}$ represent each component of the input and output matrix respectively, $x_{i}$ represents each subdivision of the epidemiological status of infected (in our case of latents $L$, infected $I$ and asymptomatic $A$ ), and $m$ is the order of the matrix $\mathscr{F}, \mathscr{V}$ (in our case $m=3$ ). Finding the Jacobian, we have:

$$
\begin{gathered}
F=\left.\frac{\partial \mathscr{F}_{i}}{\partial x_{i}}\right|_{E_{0}}=\left(\begin{array}{ccc}
\left.\frac{\partial \mathscr{F}_{L}}{\partial L}\right|_{\left(E_{0}\right)} & \left.\frac{\partial \mathscr{F}_{L}}{\partial I}\right|_{\left(E_{0}\right)} & \left.\frac{\partial \mathscr{F}_{L}}{\partial A}\right|_{\left(E_{0}\right)} \\
\left.\frac{\partial \mathscr{F}_{I}}{\partial L}\right|_{\left(E_{0}\right)} & \left.\frac{\partial \mathscr{F}_{I}}{\partial I}\right|_{\left(E_{0}\right)} & \left.\frac{\partial \mathscr{F}_{I}}{\partial A}\right|_{\left(E_{0}\right)} \\
\left.\frac{\partial \mathscr{F}_{A}}{\partial L}\right|_{\left(E_{0}\right)} & \left.\frac{\partial \mathscr{F}_{A}}{\partial I}\right|_{\left(E_{0}\right)} & \left.\frac{\partial \mathscr{F}_{A}}{\partial A}\right|_{\left(E_{0}\right)}
\end{array}\right)=\left(\begin{array}{ccc}
0 & \beta S_{0} & \beta \delta S_{0} \\
0 & 0 & 0 \\
0 & 0 & 0
\end{array}\right), \\
V=\left.\frac{\partial \mathscr{V}_{i}}{\partial x_{i}}\right|_{E_{0}}=\left(\begin{array}{lll}
\left.\frac{\partial \mathscr{V}_{L}}{\partial L}\right|_{\left(E_{0}\right)} & \left.\frac{\partial \mathscr{V}_{L}}{\partial I}\right|_{\left(E_{0}\right)} & \left.\frac{\partial \mathscr{V}_{L}}{\partial A}\right|_{\left(E_{0}\right)} \\
\left.\frac{\partial \mathscr{V}_{I}}{\partial L}\right|_{\left(E_{0}\right)} & \left.\frac{\partial \mathscr{V}_{I}}{\partial I}\right|_{\left(E_{0}\right)} & \left.\frac{\partial \mathscr{V}_{I}}{\partial A}\right|_{\left(E_{0}\right)} \\
\left.\frac{\partial \mathscr{V}_{A}}{\partial L}\right|_{\left(E_{0}\right)} & \left.\frac{\partial \mathscr{V}_{A}}{\partial I}\right|_{\left(E_{0}\right)} & \left.\frac{\partial \mathscr{V}_{A}}{\partial A}\right|_{\left(E_{0}\right)}
\end{array}\right)=\left(\begin{array}{ccc}
\kappa & 0 & 0 \\
-p \kappa & \alpha & 0 \\
-(1-p) \kappa & 0 & \eta
\end{array}\right) .
\end{gathered}
$$

The inverse matrix of $V$, given by:

$$
V^{-1}=\left(\begin{array}{ccc}
\frac{1}{\kappa} & 0 & 0 \\
\frac{p}{\alpha} & \frac{1}{\alpha} & 0 \\
\frac{-p+1}{\eta} & 0 & \frac{1}{\eta}
\end{array}\right)
$$

Let the matrix product $K=F V^{-1}$ be named next generation matrix:

$$
K=\left(\begin{array}{ccc}
0 & \beta S_{0} & \beta \delta S_{0} \\
0 & 0 & 0 \\
0 & 0 & 0
\end{array}\right)\left(\begin{array}{ccc}
\frac{1}{\kappa} & 0 & 0 \\
\frac{p}{\alpha} & \frac{1}{\alpha} & 0 \\
\frac{-p+1}{\eta} & 0 & \frac{1}{\eta}
\end{array}\right)=\left(\begin{array}{ccc}
\frac{\alpha \beta \delta S_{0}(1-p)+\eta \beta p S_{0}}{\alpha \eta} & \frac{\beta S_{0}}{\alpha} & \frac{\beta \delta S_{0}}{\eta} \\
0 & 0 & 0 \\
0 & 0 & 0
\end{array}\right) .
$$

The basic playback number is defined as the spectral radius of the next generation matrix [25, 3] i.e., as the higher of the eigenvalues or dominant eigenvalue of the next generation matrix [11]:

$$
\mathscr{R}_{0}=\rho\left(F V^{-1}\right)=\rho(K) .
$$

Calculating the eigenvalues as the roots of the characteristic polynomial associated with the next generation matrix, we have:

$$
\begin{aligned}
& \operatorname{det}\left(K-\lambda I_{3}\right)=0 \\
& \operatorname{det}\left(\begin{array}{ccc}
\frac{\alpha \beta \delta S_{0}(1-p)+\eta \beta p S_{0}}{\alpha \eta}-\lambda & \frac{\beta S_{0}}{\alpha} & \frac{\beta \delta S_{0}}{\eta} \\
0 & 0-\lambda & 0 \\
0 & 0 & 0-\lambda
\end{array}\right)=0 \\
& -\lambda^{2}\left(\lambda-S_{0} \beta\left(\frac{\alpha \delta(1-p)+\eta p}{\alpha \eta}\right)\right)=0 \Leftrightarrow \lambda_{1}=\lambda_{2}=0 \quad \vee \quad \lambda_{3}=S_{0} \beta\left(\frac{\alpha \delta(1-p)+\eta p}{\alpha \eta}\right) .
\end{aligned}
$$

It follows that $\mathscr{R}_{0}$, takes the form of:

$$
\mathscr{R}_{0}=S_{0} \beta\left(\frac{\delta(1-p)}{\eta}+\frac{p}{\alpha}\right) .
$$

2.3.3. Local stability. We analyze the stability of the disease-free equilibrium point. The last equation of the system (2.1) can be omitted because its dynamics does not depend of itself. Linearizing, we get the Jacobian matrix:

$$
J=\left(\begin{array}{cccc}
-\beta(I+\delta A) & 0 & -\beta S & -\beta \delta S \\
\beta(I+\delta A) & -\kappa & \beta S & \beta \delta S \\
0 & p \kappa & -\alpha & 0 \\
0 & (1-p) \kappa & 0 & -\eta
\end{array}\right),
$$


thus, analyzing the disease-free point stability

$$
J\left(E_{0}\right)=\left(\begin{array}{cccc}
0 & 0 & -\beta S_{0} & -\beta \delta S_{0} \\
0 & -\kappa & \beta S_{0} & \beta \delta S_{0} \\
0 & p \kappa & -\alpha & 0 \\
0 & (1-p) \kappa & 0 & -\eta
\end{array}\right),
$$

whose characteristic equation is the next:

$$
\lambda\left(\lambda^{3}+c_{2} \lambda^{2}+c_{1} \lambda+c_{0}\right)=0,
$$

where:

$c_{2}=\eta+\alpha+\kappa, \quad c_{1}=\left(\beta \delta(p-1) S_{0}+\eta+\alpha\right) \kappa-p \kappa \beta S_{0}+\eta \alpha, c_{0}=\left(\beta \delta(p-1) S_{0}+\eta\right) \alpha \kappa-\beta \eta p \kappa S_{0}$

By the Routh-Hurwitz criterion, we can assure that the characteristic polynomial has roots with negative real parts only if $c_{2}>0, c_{1}>0, c_{0}>0$ (from this condition it follows that $\mathscr{R}_{0}<1$ ) and $c_{1} c_{2}>c_{0}$. Although a condition was obtained to study the central manifold (due to $\exists \lambda=0$ ) for this point, we will not focus on the stability of $E_{0}$.

\section{SLIIAR model.}

3.1. Hypothesis. The proposed model consists of the following considerations:

- This model is a variation of the SLIIAR model based on the stratification of those infected with symptoms in two compartments $\left(I_{1}, I_{2}\right)$, we considered vital dynamics and also deaths due to disease.

- It is considered that the two sub-populations of symptomatic infectious individuals can spread the disease with equal intensity.

- Two types of treatment are considered: vaccination and symptomatic treatment.

- It is considered the existence of latent individuals $(L)$, which are infected but do not transmit the disease.

- Asymptomatic individuals $(A)$ are considered to be able to transmit the disease. In addition, the transmission is attenuated by an $\xi$ attenuation rate.

- It is considered that a symptomatic infected person can revert to a previous state of less severity by the action of a symptomatological treatment (medicines and/or palliative care), and does so with a rate of reversion $\varepsilon$, which is the inverse of the days needed for symptoms to decrease in severity.

- It is considered that there is an immunization intervention, with a vaccination rate $\kappa$.

The flow diagram of the model is as follows:

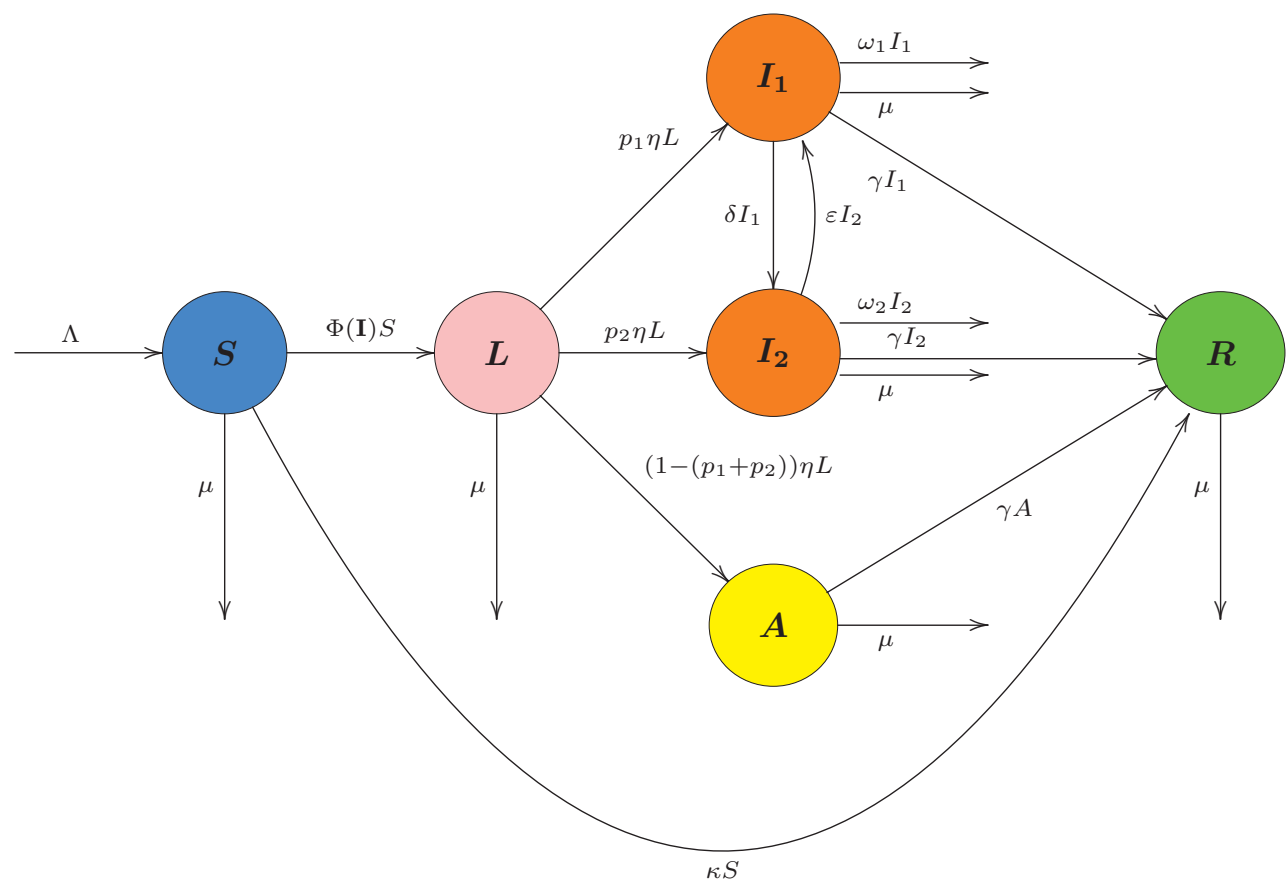

Figure 3.1: Flow diagram from SLIIAR model 
Considering the incidence as $\Phi(\mathbf{I}) S$, and the force of infection is given by:

$$
\Phi(\mathbf{I})=\beta\left(I_{1}+I_{2}+\xi A\right), \mathbf{I}=\left(I_{1} ; I_{2} ; A\right), \Phi: \mathbb{R}_{+}^{3} \rightarrow \mathbb{R}_{+} .
$$

Where in addition to the previous variables, we can mention the next:

- $I_{1}(t)=I_{1}$, represents the population of infectious individuals with mild symptoms within the population in an instant $t$.

- $I_{2}(t)=I_{2}$, represents the population of infectious individuals with severe symptoms within the population in an instant $t$.

- $\Lambda$, represents the recruitment rate.

- $\beta$, represents the transmission rate.

- $\mu$, represents the mortality rate independent of the disease.

- $\xi, 0 \leq \xi \leq 1$, is the rate of attenuation of transmission for asymptomatic persons.

- $\eta$, is the rate of transition from latent to symptomatic or asymptomatic infected.

- $p_{i}, \forall i=1,2$, is the proportion or probability that the latent population becomes one of the possible infectious states (symptomatic and asymptomatic).

- $\delta$, is the transition rate of a symptomatic mild to severe infected individual.

- $\varepsilon$, is the rate of symptom reversal between the different levels of symptomatic infected.

- $\gamma$, is the rate of infection recovery for those infected (symptomatic and asymptomatic).

- $\omega_{i}, \forall i=1,2$, is the lethality rate of the infection for symptomatic infected.

- $\kappa$, is the constant vaccination rate.

The model can be formalized as a system of ordinary differential equations:

$$
\left\{\begin{array}{lll}
\frac{d S}{d t}=\Lambda-\beta\left(I_{1}+I_{2}+\xi A\right) S-(\kappa+\mu) S, & S(0)=S_{0}>0 \\
\frac{d L}{d t}=\beta\left(I_{1}+I_{2}+\xi A\right) S-(\eta+\mu) L & L(0)=L_{0} \geq 0 \\
\frac{d I_{1}}{d t}=p_{1} \eta L+\varepsilon I_{2}-\left(\delta+\gamma+\omega_{1}+\mu\right) I_{1}, & I_{1}(0)=I_{1_{0}} \geq 0 \\
\frac{d I_{2}}{d t}=p_{2} \eta L+\delta I_{1}-\left(\varepsilon+\gamma+\omega_{2}+\mu\right) I_{2}, & I_{2}(0)=I_{2_{0}} \geq 0 \\
\frac{d A}{d t}=\left(1-p_{1}-p_{2}\right) \eta L-(\gamma+\mu) A, & & A(0)=A_{0} \geq 0 \\
\frac{d R}{d t}=\gamma I_{1}+\gamma I_{2}+\gamma A+\kappa S-\mu R, & & R(0)=R_{0} \geq 0 \\
\left(\Lambda, \beta, \xi, \kappa, \mu, \eta, p_{1}, p_{2}, \varepsilon, \delta, \gamma, \omega_{1}, \omega_{2}\right) \in \mathbb{R}_{+}^{13} . &
\end{array}\right.
$$

\subsection{Basic qualitative properties.}

3.2.1. Positivity. Lemma 3.1. The system given by (3.1), with initial conditions $S(0)>0, L(0) \geq$ $0, I_{1}(0) \geq 0, I_{2}(0) \geq 0, A(0) \geq 0, R(0) \geq 0$ have non-negative solutions $S(t), L(t), I_{1}(t), I_{2}(t), A(t), R(t), \forall t \geq$ 0 .

Proof: The proof is given by contradiction.

- If exists a negative solution for $S(t)$ with $S(0)>0$, so there must exist a

$$
t_{1}=\sup \left\{0 \leq t \leq t_{1} \mid S(t) \geq 0, L(t) \geq 0, I_{1}(t) \geq 0, I_{2}(t) \geq 0, A(t) \geq 0, R(t) \geq 0\right\},
$$

such that

$$
S\left(t_{1}\right)=0, S^{\prime}\left(t_{1}\right)<0
$$

but from the first equation (3.1), we have that

$$
S^{\prime}\left(t_{1}\right)=\Lambda>0
$$

and this result means that $S(t) \geq 0, \forall t \geq 0$, which is a contradiction with the hypothesis, then $S(t) \geq 0, \forall t \geq 0$.

- In the case of $L(t)$ with $L(0) \geq 0$, if $L(t)<0$ so there must exist a

$$
t_{2}=\sup \left\{0 \leq t \leq t_{2} \mid S(t) \geq 0, L(t) \geq 0, I_{1}(t) \geq 0, I_{2}(t) \geq 0, A(t) \geq 0, R(t) \geq 0\right\}
$$


such that

$$
L\left(t_{2}\right)=0, L^{\prime}\left(t_{2}\right)<0,
$$

from the second equation (3.1), we have that:

$$
L^{\prime}\left(t_{2}\right) \geq 0, \forall t \geq 0 \text {. }
$$

It means that $L(t) \geq 0, \forall t \geq 0$, in particular $L(t)>0, \forall t>0$ which is a contradiction with the hypothesis in this case, so we can conclude that $L(t) \geq 0, \forall t \geq 0$.

- Following the same analysis for the other populations, we have that:

$$
S(t), L(t), I_{1}(t), I_{2}(t), A(t), R(t)>0, \forall t \geq 0 .
$$

3.2.2. Boundedness. Lemma 3.2. The system (3.1) with initial conditions $S(0)>0, L(0) \geq 0, I_{1}(0) \geq$ $0, I_{2}(0) \geq 0, A(0) \geq 0, R(0) \geq 0$ is positively invariant in the feasible region $\Omega$, defined by:

$$
\Omega=\left\{\left(S(t), L(t), I_{1}(t), I_{2}(t), A(t), R(t)\right) \in \mathbb{R}_{+}^{6}: N(t) \leq \frac{\Lambda}{\mu}\right\} .
$$

Proof: Let the ordinary differential equations system be (3.1) and adding the simplified system,we have:

$$
\frac{d S}{d t}+\frac{d L}{d t}+\frac{d L_{1}}{d t}+\frac{d L_{2}}{d t}+\frac{d A}{d t}+\frac{d R}{d t}=\Lambda-\mu\left(S+L+I_{1}+I_{2}+A+R\right)-\omega_{1} I_{1}-\omega_{2} I_{2},
$$

as $N(t)=S(t)+L(t)+I_{1}(t)+I_{2}(t)+A(t)+R(t)$, then:

$$
\frac{d N}{d t}=\Lambda-\mu N-\omega_{1} I_{1}-\omega_{2} I_{2} \leq \Lambda-\mu N,
$$

solving the differential inequality

$$
\begin{gathered}
\frac{d N}{d t} \leq \Lambda-\mu N \\
\frac{d\left(N e^{\mu t}\right)}{d t} \leq \Lambda e^{\mu t}
\end{gathered}
$$

by integration we obtain

$$
\begin{gathered}
N(t) \leq e^{-\mu t}\left(\int e^{\mu t} \Lambda d t+C\right) \\
N(t) \leq \frac{\Lambda}{\mu}+C e^{-\mu t} .
\end{gathered}
$$

We notice that as $N(t)=S(t)+L(t)+I_{1}(t)+I_{2}(t)+A(t)+R(t)$ and the initial conditions $S(0) \geq$ $0, L(0) \geq 0, I_{1}(0) \geq 0, I_{2}(0) \geq 0, A(0) \geq 0, R(0) \geq 0$, so that $N(0) \geq 0$ and $N(t) \geq 0$ by the previous Lemma. In order to determine the integration constant value, in $t=0$ we have that $N(0)$ (that represents the total initial population)

$$
\begin{aligned}
& N(0) \leq \frac{\Lambda}{\mu}+C \\
& N(0)-\frac{\Lambda}{\mu} \leq C,
\end{aligned}
$$

thus,

$$
N(t) \leq \frac{\Lambda}{\mu}+e^{-\mu t}\left(N(0)-\frac{\Lambda}{\mu}\right),
$$

so, we have that when $t \longrightarrow \infty$ :

$$
\lim _{t \rightarrow \infty} N(t) \leq \frac{\Lambda}{\mu}
$$


3.3. Qualitative Analysis. The equilibrium points can be found by setting each of the equations of the system 3.1 equal to zero.

3.3.1. Disease-free equilibrium point. Theorem 3.1 (Existence of disease-free equilibrium point). The disease-free equilibrium point $E_{0}$ always exists for the system (3.1).

Proof: We proceed to determine the disease-free equilibrium point, considering the steady state of the system (3.1), when $I_{1}=I_{2}=A=0$, so we found that $L=0, S=\frac{\Lambda}{\kappa+\mu}, R=\frac{\kappa \Lambda}{\mu(\kappa+\mu)}$. Moreover, the whole parameters are positive, so the positively of the of the following point is assured:

$$
E_{0}=\left(\frac{\Lambda}{\kappa+\mu}, 0,0,0,0, \frac{\kappa \Lambda}{\mu(\kappa+\mu)}\right)
$$

3.3.2. Calculating $R_{0}$ using the next generation matrix. Following the methodology of AmayaMuvdi [3] used previously, we will evaluate the subsystem of infected compartments:

$$
\left\{\begin{aligned}
\frac{d L}{d t} & ==\beta\left(I_{1}+I_{2}+\xi A\right) S-(\eta+\mu) L \\
\frac{d I_{1}}{d t} & =p_{1} \eta L+\varepsilon I_{2}-\left(\delta+\gamma+\omega_{1}+\mu\right) I_{1} \\
\frac{d I_{2}}{d t} & =p_{2} \eta L+\delta I_{1}-\left(\varepsilon+\gamma+\omega_{2}+\mu\right) I_{2} \\
\frac{d A}{d t} & =\left(1-p_{1}-p_{2}\right) \eta L-(\gamma+\mu) A .
\end{aligned}\right.
$$

From this subsystem, we will form the matrix of new infections appearance rates $\mathscr{F}_{\left(L, I_{1}, I_{2}, A\right)}$, and the matrix of transfer rates $\mathscr{V}_{\left(L, I_{1}, I_{2}, A\right)}$, which in this case take the form:

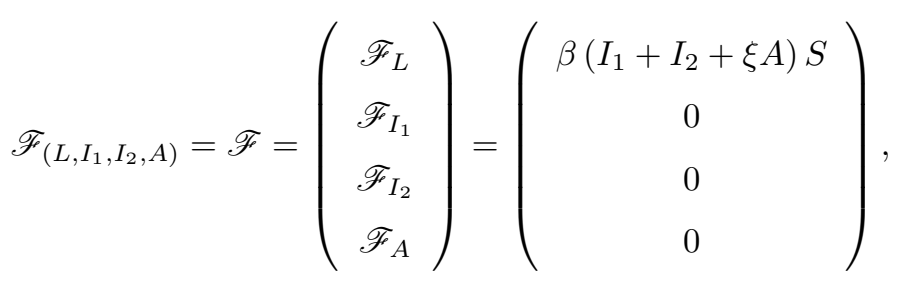

$$
\begin{aligned}
& \mathscr{V}_{\left(L, I_{1}, I_{2}, A\right)}^{+}=\mathscr{V}^{+}=\left(\begin{array}{c}
\mathscr{V}_{L}^{+} \\
\mathscr{V}_{I_{1}}^{+} \\
\mathscr{V}_{I_{2}}^{+} \\
\mathscr{V}_{A}^{+}
\end{array}\right)=\left(\begin{array}{c}
0 \\
p_{1} \eta L+\varepsilon I_{2} \\
p_{2} \eta L+\delta I_{1} \\
\left(1-p_{1}-p_{2}\right) \eta L
\end{array}\right) \\
& \mathscr{V}_{\left(L, I_{1}, I_{2}, A\right)}^{-}=\mathscr{V}^{-}=\left(\begin{array}{c}
\mathscr{V}_{L}^{-} \\
\mathscr{V}_{I_{1}}^{-} \\
\mathscr{V}_{I_{2}}^{-} \\
\mathscr{V}_{A}^{-}
\end{array}\right)=\left(\begin{array}{c}
(\eta+\mu) L \\
\left(\delta+\gamma+\omega_{1}+\mu\right) I_{1} \\
\left(\varepsilon+\gamma+\omega_{2}+\mu\right) I_{2} \\
(\gamma+\mu) A
\end{array}\right) \\
& \mathscr{V}_{\left(L, I_{1}, I_{2}, A\right)}=\mathscr{V}=\mathscr{V}_{\left(L, I_{1}, I_{2}, A\right)}^{-}-\mathscr{V}_{\left(L, I_{1}, I_{2}, A\right)}^{+}=\mathscr{V}^{-}-\mathscr{V}^{+}=\left(\begin{array}{c}
(\eta+\mu) L \\
\left(\delta+\gamma+\omega_{1}+\mu\right) I_{1}-p_{1} \eta L-\varepsilon I_{2} \\
\left(\varepsilon+\gamma+\omega_{2}+\mu\right) I_{2}-p_{2} \eta L-\delta I_{1} \\
(\gamma+\mu) A-\left(1-p_{1}-p_{2}\right) \eta L
\end{array}\right)
\end{aligned}
$$

We proceed to calculate the Jacobian matrix of $\mathscr{V}$ and $\mathscr{F}$, evaluating them at the disease-free equilibrium point for our case $E_{0}=\left(\frac{\Lambda}{\kappa+\mu}, 0,0,0,0, \frac{\kappa \Lambda}{\mu(\kappa+\mu)}\right)$ : 


$$
\begin{aligned}
& F=\left.\frac{\partial \mathscr{F}_{i}}{\partial x_{i}}\right|_{E_{0}}=\left(\begin{array}{cccc}
\left.\frac{\partial \mathscr{F}_{L}}{\partial L}\right|_{\left(E_{0}\right)} & \left.\frac{\partial \mathscr{F}_{L}}{\partial I_{1}}\right|_{\left(E_{0}\right)} & \left.\frac{\partial \mathscr{F}_{L}}{\partial I_{2}}\right|_{\left(E_{0}\right)} & \left.\frac{\partial \mathscr{F}_{L}}{\partial A}\right|_{\left(E_{0}\right)} \\
\left.\frac{\partial \mathscr{F}_{1}}{\partial L}\right|_{\left(E_{0}\right)} & \left.\frac{\partial \mathscr{F}_{I_{1}}}{\partial I_{1}}\right|_{\left(E_{0}\right)} & \left.\frac{\partial \mathscr{F}_{I_{1}}}{\partial I_{2}}\right|_{\left(E_{0}\right)} & \left.\frac{\partial \mathscr{F}_{1}}{\partial A}\right|_{\left(E_{0}\right)} \\
\left.\frac{\partial \mathscr{F}_{I_{2}}}{\partial L}\right|_{\left(E_{0}\right)} & \left.\frac{\partial \mathscr{F}_{I_{2}}}{\partial I_{1}}\right|_{\left(E_{0}\right)} & \left.\frac{\partial \mathscr{F}_{I_{2}}}{\partial I_{2}}\right|_{\left(E_{0}\right)} & \left.\frac{\partial \mathscr{F}_{I_{2}}}{\partial A}\right|_{\left(E_{0}\right)} \\
\left.\frac{\partial \mathscr{F}_{A}}{\partial L}\right|_{\left(E_{0}\right)} & \left.\frac{\partial \mathscr{F}_{A}}{\partial I_{1}}\right|_{\left(E_{0}\right)} & \left.\frac{\partial \mathscr{F}_{A}}{\partial I_{2}}\right|_{\left(E_{0}\right)} & \left.\frac{\partial \mathscr{F}_{A}}{\partial A}\right|_{\left(E_{0}\right)}
\end{array}\right) \\
& F=\left(\begin{array}{cccc}
0 & \frac{\beta \Lambda}{\kappa+\mu} & \frac{\beta \Lambda}{\kappa+\mu} & \frac{\beta \xi \Lambda}{\kappa+\mu} \\
0 & 0 & 0 & 0 \\
0 & 0 & 0 & 0 \\
0 & 0 & 0 & 0
\end{array}\right) \\
& V=\left.\frac{\partial \mathscr{V}_{i}}{\partial x_{i}}\right|_{E_{0}}=\left(\begin{array}{cccc}
\left.\frac{\partial \mathscr{V}_{L}}{\partial L}\right|_{\left(E_{0}\right)} & \left.\frac{\partial \mathscr{V}_{L}}{\partial I_{1}}\right|_{\left(E_{0}\right)} & \left.\frac{\partial \mathscr{V}_{L}}{\partial I_{2}}\right|_{\left(E_{0}\right)} & \left.\frac{\partial \mathscr{V}_{L}}{\partial A}\right|_{\left(E_{0}\right)} \\
\left.\frac{\partial \mathscr{V}_{I_{1}}}{\partial L}\right|_{\left(E_{0}\right)} & \left.\frac{\partial \mathscr{V}_{I_{1}}}{\partial I_{1}}\right|_{\left(E_{0}\right)} & \left.\frac{\partial \mathscr{V}_{I_{1}}}{\partial I_{2}}\right|_{\left(E_{0}\right)} & \left.\frac{\partial \mathscr{V}_{I_{1}}}{\partial A}\right|_{\left(E_{0}\right)} \\
\left.\frac{\partial \mathscr{V}_{I_{2}}}{\partial L}\right|_{\left(E_{0}\right)} & \left.\frac{\partial \mathscr{V}_{I_{2}}}{\partial I_{1}}\right|_{\left(E_{0}\right)} & \left.\frac{\partial \mathscr{V}_{I_{2}}}{\partial I_{2}}\right|_{\left(E_{0}\right)} & \left.\frac{\partial \mathscr{V}_{I_{2}}}{\partial A}\right|_{\left(E_{0}\right)} \\
\left.\frac{\partial \mathscr{V}_{A}}{\partial L}\right|_{\left(E_{0}\right)} & \left.\frac{\partial \mathscr{V}_{A}}{\partial I_{1}}\right|_{\left(E_{0}\right)} & \left.\frac{\partial \mathscr{V}_{A}}{\partial I_{2}}\right|_{\left(E_{0}\right)} & \left.\frac{\partial \mathscr{V}_{A}}{\partial A}\right|_{\left(E_{0}\right)}
\end{array}\right) \\
& V=\left(\begin{array}{cccc}
\eta+\mu & 0 & 0 & 0 \\
-p_{1} \eta & \delta+\gamma+\omega_{1}+\mu & -\varepsilon & 0 \\
-p_{2} \eta & -\delta & \varepsilon+\gamma+\omega_{2}+\mu & 0 \\
-\left(1-p_{1}-p_{2}\right) \eta & 0 & 0 & \gamma+\mu
\end{array}\right) .
\end{aligned}
$$

It is necessary to find the inverse matrix of $V$ and then calculate the matrix product $K=F V^{-1}$ called next generation matrix. The basic reproduction number is defined as the spectral radius of the next generation matrix $[25,3]$ that is, as the largest of the eigenvalues or dominant eigenvalue of the next generation matrix [11]:

$$
\mathscr{R}_{0}=\rho\left(F V^{-1}\right)=\rho(K)
$$

Calculating all the steps, we obtain the following basic reproductive number:

$$
\begin{gathered}
\mathscr{R}_{0}=\eta\left\{\frac{\left(1-p_{1}-p_{2}\right) \Lambda\left[\mu^{2}+\left(\varepsilon+\omega_{1}+\omega_{2}+\delta+2 \gamma\right) \mu+\gamma^{2}+\left(\varepsilon+\omega_{1}+\omega_{2}+\delta\right) \gamma+\left(\varepsilon+\omega_{2}\right) \omega_{1}+\omega_{2} \delta\right] \beta \xi}{(\gamma+\mu)\left[\mu^{2}+\left(\varepsilon+\omega_{1}+\omega_{2}+\delta+2 \gamma\right) \mu+\gamma^{2}+\left(\varepsilon+\omega_{1}+\omega_{2}+\delta\right) \gamma+\left(\varepsilon+\omega_{2}\right) \omega_{1}+\omega_{2} \delta\right](\eta+\mu)(\kappa+\mu)}\right. \\
\left.+\frac{(\gamma+\mu) \beta \Lambda\left[\left(p_{1}+p_{2}\right) \mu+\left(p_{1}+p_{2}\right) \gamma+\left(\varepsilon+\omega_{2}+\delta\right) p_{1}+p_{2}\left(\varepsilon+\omega_{1}+\delta\right)\right]}{(\gamma+\mu)\left[\mu^{2}+\left(\varepsilon+\omega_{1}+\omega_{2}+\delta+2 \gamma\right) \mu+\gamma^{2}+\left(\varepsilon+\omega_{1}+\omega_{2}+\delta\right) \gamma+\left(\varepsilon+\omega_{2}\right) \omega_{1}+\omega_{2} \delta\right](\eta+\mu)(\kappa+\mu)}\right\}, \\
\mathscr{R}_{0}=\frac{\beta \eta \Lambda}{(\eta+\mu)(\kappa+\mu)}\left\{\frac{p_{1}\left(\varepsilon+\delta+\mu+\gamma+\omega_{2}\right)}{\left[\left(\gamma+\mu+\omega_{1}\right)\left(\gamma+\mu+\omega_{2}+\varepsilon\right)+\delta\left(\gamma+\mu+\omega_{2}\right)\right]}\right. \\
\left.+\frac{p_{2}\left(\varepsilon+\delta+\mu+\gamma+\omega_{1}\right)}{\left[\left(\gamma+\mu+\omega_{1}\right)\left(\gamma+\mu+\omega_{2}+\varepsilon\right)+\delta\left(\gamma+\mu+\omega_{2}\right)\right]}+\frac{\xi\left(1-p_{1}-p_{2}\right)}{(\gamma+\mu)}\right\} .
\end{gathered}
$$

3.3.3. Endemic equilibrium point. Theorem 3.2 (Existence of endemic equilibrium point). The endemic equilibrium point:

$$
E_{1}=\left(S^{*}, L^{*}, I_{1}^{*}, I_{2}^{*}, A^{*}, R^{*}\right)
$$

exists for the system (3.1) only if $\mathscr{R}_{0}>1$. Where $S^{*}, L^{*}, I_{1}^{*}, I_{2}^{*}, A^{*}, R^{*}>0$.

Proof: Solving the system (3.1), we get another equilibrium point:

$$
E_{1}=\left(S^{*}, L^{*}, I_{1}^{*}, I_{2}^{*}, A^{*}, R^{*}\right)
$$


where

$$
\begin{aligned}
S^{*} & =\frac{\eta+\mu}{\beta \eta\left\{\frac{\left(\varepsilon+\delta+\mu+\gamma+\omega_{2}\right) p_{1}}{\left[\left(\gamma+\mu+\omega_{1}\right)\left(\gamma+\mu+\omega_{2}+\varepsilon\right)+\delta\left(\gamma+\mu+\omega_{2}\right)\right]}+\frac{\left(\varepsilon+\delta+\mu+\gamma+\omega_{1}\right) p_{2}}{\left[\left(\gamma+\mu+\omega_{1}\right)\left(\gamma+\mu+\omega_{2}+\varepsilon\right)+\delta\left(\gamma+\mu+\omega_{2}\right)\right]}+\frac{\xi\left(1-p_{1}-p_{2}\right)}{(\gamma+\mu)}\right\}} \\
L^{*} & =\frac{\Lambda-(\kappa+\mu) S^{*}}{\eta+\mu} \\
I_{1}^{*} & =\left\{\frac{\left(\varepsilon+\gamma+\omega_{2}+\mu\right) p_{1}+\varepsilon p_{2}}{\left[\left(\gamma+\mu+\omega_{1}\right)\left(\gamma+\mu+\omega_{2}+\varepsilon\right)+\delta\left(\gamma+\mu+\omega_{2}\right)\right]}\right\} \eta L^{*} \\
I_{2}^{*} & =\left\{\frac{\left(\delta+\gamma+\omega_{1}+\mu\right) p_{2}+\delta p_{1}}{\left[\left(\gamma+\mu+\omega_{1}\right)\left(\gamma+\mu+\omega_{2}+\varepsilon\right)+\delta\left(\gamma+\mu+\omega_{2}\right)\right]}\right\} \eta L^{*} \\
A^{*} & =\frac{\left(1-p_{1}-p_{2}\right)}{\gamma+\mu} \eta L^{*} \\
R^{*} & =\frac{\gamma\left(I_{1}^{*}+I_{2}^{*}+A^{*}\right)+\kappa S^{*}}{\mu}
\end{aligned}
$$

using (3.2) and (3.3) we deduce the equivalence

$$
S^{*}=\frac{\Lambda}{(\kappa+\mu) \mathscr{R}_{0}},
$$

furthermore, the equation (3.4) must satisfy the following inequality to be positive

$$
\Lambda>(\kappa+\mu) S^{*},
$$

thus, as $S^{*}(\kappa+\mu)=\frac{\Lambda}{\mathscr{R}_{0}}$, we have

$$
\Lambda>\frac{\Lambda}{\mathscr{R}_{0}} \Leftrightarrow \mathscr{R}_{0}>1
$$

3.3.4. Local stability for disease-free equilibrium point. Linearizing the system (3.1), we get the Jacobian matrix:

$$
J=\left(\begin{array}{cccccc}
-\beta\left(I_{1}+I_{2}+\xi A\right)-(\kappa+\mu) & 0 & -\beta S & -\beta S & -\beta \xi S & 0 \\
\beta\left(I_{1}+I_{2}+\xi A\right) & -(\eta+\mu) & \beta S & \beta S & \beta \xi S & 0 \\
0 & p_{1} \eta & -\left(\delta+\gamma+\omega_{1}+\mu\right) & \varepsilon & 0 & 0 \\
0 & p_{2} \eta & \delta & -\left(\varepsilon+\gamma+\omega_{2}+\mu\right) & 0 & 0 \\
0 & \left(1-p_{1}-p_{2}\right) \eta & 0 & 0 & -(\gamma+\mu) & 0 \\
\kappa & 0 & \gamma & \gamma & \gamma & -\mu
\end{array}\right) .
$$

Considering the disease-free equilibrium point $E_{0}=\left(\frac{\Lambda}{\kappa+\mu}, 0,0,0,0, \frac{\kappa \Lambda}{\mu(\kappa+\mu)}\right)$, we can evaluate this point in the Jacobian matrix:

$$
J\left(E_{0}\right)=\left(\begin{array}{cccccc}
-(\kappa+\mu) & 0 & -\beta\left(\frac{\Lambda}{\kappa+\mu}\right) & -\beta\left(\frac{\Lambda}{\kappa+\mu}\right) & -\beta \xi\left(\frac{\Lambda}{\kappa+\mu}\right) & 0 \\
0 & -(\eta+\mu) & \beta\left(\frac{\Lambda}{\kappa+\mu}\right) & \beta\left(\frac{\Lambda}{\kappa+\mu}\right) & \beta \xi\left(\frac{\Lambda}{\kappa+\mu}\right) & 0 \\
0 & p_{1} \eta & -\left(\delta+\gamma+\omega_{1}+\mu\right) & \varepsilon & 0 & 0 \\
0 & p_{2} \eta & \delta & -\left(\varepsilon+\gamma+\omega_{2}+\mu\right) & 0 & 0 \\
0 & \left(1-p_{1}-p_{2}\right) \eta & 0 & 0 & -(\gamma+\mu) & 0 \\
\kappa & 0 & \gamma & \gamma & \gamma & -\mu
\end{array}\right) .
$$

Let

$$
A=\kappa+\mu, \quad, B=\eta+\mu, \quad C=\delta+\gamma+\omega_{1}+\mu,
$$




$$
D=\varepsilon+\gamma+\omega_{2}+\mu, \quad E=\gamma+\mu, \quad F=\frac{\beta \Lambda}{\kappa+\mu}, \quad p=1-p_{1}-p_{2},
$$

where the characteristic polynomial is given by

$$
\begin{aligned}
p(\lambda)= & (\lambda+\mu)(\lambda+A)\left(\lambda^{4}+(B+C+D+E) \lambda^{3}+\left((B+C+D) E-F\left(p_{1}+p_{2}\right) \eta+(B+D) C\right.\right. \\
& +D B-p \eta F \xi-\delta \varepsilon) \lambda^{2}+\left(\left(-F\left(p_{1}+p_{2}\right) \eta+(B+D) C+D B-\delta \varepsilon\right) E-F\left(C p_{2}+D p_{1}+\right.\right. \\
& \left.\left.\delta p_{1}+\varepsilon p_{2}\right) \eta+(D B-p \eta F \xi) C-B \delta \varepsilon-D F \xi p \eta\right) \lambda+\left(-F\left(C p_{2}+D p_{1}+\delta p_{1}+\varepsilon p_{2}\right) \eta-\right. \\
& B(-C D+\delta \varepsilon)) E+p \eta F \xi(-C D+\delta \varepsilon))=0 .
\end{aligned}
$$

We denote the coefficients of the fourth-order polynomial as follow:

$$
\begin{aligned}
& a_{4}=1, a_{3}=B+C+D+E, a_{2}=(B+C+D) E-F \eta\left(p_{1}+p_{2}\right)+(B+D) C-\delta \varepsilon-p \eta \xi F+D B \\
& a_{1}=\left[(B+D) C-F \eta\left(p_{1}+p_{2}\right)-\delta \varepsilon+B D\right] E-F \eta\left(C p_{2}+D p_{1}+\delta p_{1}+\varepsilon p_{2}\right)+(B D-F \eta p \xi) C \\
& \quad-B \delta \varepsilon-F D \xi \eta p \\
& \quad a_{0}=\left[-F \eta\left(C p_{2}+D p_{1}+\delta p_{1}+\varepsilon p_{2}\right)-B(\delta \varepsilon-D C)\right] E+F p \eta \xi(\delta \varepsilon-D C),
\end{aligned}
$$

thus

$$
p(\lambda)=(\lambda+A)(\lambda+\mu)\left(a_{4} \lambda^{4}+a_{3} \lambda^{3}+a_{2} \lambda^{2}+a_{1} \lambda+a_{0}\right)=0 .
$$

By the Routh-Hurwitz criterion, the disease-free point is locally asymptotically stable only if the eigenvalues of the Jacobian matrix have negative real parts. Then, the first two roots of the polynomial are $\lambda_{1}=$ $-A<0, \lambda_{2}=-\mu<0$ and the other roots for the fourth-order polynomial have real negative parts if the coefficients of the characteristic polynomial satisfied: $a_{0}>0, a_{1}>0, a_{2}>0, a_{3}>0, a_{4}>0, a_{3} a_{2}>a_{4} a_{1}$ and $a_{3} a_{2} a_{1}>a_{4} a_{1}^{2}+a_{3}^{2} a_{0}$. From $a_{0}>0$, it follows that $\mathscr{R}_{0}<1$. We get the following result:

Theorem 3.3 (Local stability of disease-free equilibrium point). The disease-free equilibrium point $E_{0}$ of the system 3.1 is locally asymptotically stable only if $a_{i}>0\left(\mathscr{R}_{0}<1\right), \forall i=1, \cdots, 4, a_{3} a_{2}>a_{4} a_{1}$ and $a_{3} a_{2} a_{1}>a_{4} a_{1}^{2}+a_{3}^{2} a_{0}$.

3.3.5. Local stability for endemic equilibrium point. Evaluating the endemic equilibrium point $E_{1}=\left(S^{*}, L^{*}, I_{1}^{*}, I_{2}^{*}, A^{*}, R^{*}\right)$ in the Jacobian matrix:

$$
J\left(E_{1}\right)=\left(\begin{array}{cccccc}
-\beta\left(I_{1}^{*}+I_{2}^{*}+\xi A^{*}\right)-(\kappa+\mu) & 0 & -\beta S^{*} & -\beta S^{*} & -\beta \xi S^{*} & 0 \\
\beta\left(I_{1}^{*}+I_{2}^{*}+\xi A^{*}\right) & -(\eta+\mu) & \beta S^{*} & \beta S^{*} & \beta \xi S^{*} & 0 \\
0 & p_{1} \eta & -\left(\delta+\gamma+\omega_{1}+\mu\right) & \varepsilon & 0 & 0 \\
0 & p_{2} \eta & \delta & -\left(\varepsilon+\gamma+\omega_{2}+\mu\right) & 0 & 0 \\
0 & \left(1-p_{1}-p_{2}\right) \eta & 0 & 0 & -(\gamma+\mu) & 0 \\
\kappa & 0 & \gamma & \gamma & \gamma & -\mu
\end{array}\right) .
$$

Let

$$
\begin{gathered}
A=\kappa+\mu, \quad B=\eta+\mu, \quad C=\delta+\gamma+\omega_{1}+\mu, \quad D=\varepsilon+\gamma+\omega_{2}+\mu, \\
E=\gamma+\mu, \quad F=\beta\left(I_{1}^{*}+I_{2}^{*}+\xi A^{*}\right), \quad G=\beta S^{*}, \quad p=1-p_{1}-p_{2} .
\end{gathered}
$$

So that, the characteristic equation is the following:

$$
p(\lambda)=(\lambda+\mu)\left(\lambda^{5}+b_{4} \lambda^{4}+b_{3} \lambda^{3}+b_{2} \lambda^{2}+b_{1} \lambda+b_{0}\right),
$$

where:

$$
\begin{aligned}
b_{4}= & A+B+C+D+E+F \\
b_{3}= & E(D+C+B+F+A)+(B+C+D) A+(B+D+F) C+(B+F) D+B F- \\
& G\left(p_{1}+p_{2}\right) \eta-G \xi p \eta-\delta \varepsilon \\
b_{2}= & \left((B+C+D) A+(B+D+F) C+(B+F) D+B F-G\left(p_{1}+p_{2}\right) \eta-\delta \varepsilon\right) E+ \\
& \left((B+D) C+B D-G\left(p_{1}+p_{2}\right) \eta-G \xi p \eta-\delta \varepsilon\right) A+\left((B+F) D-G \eta p_{2}-G \xi p \eta+B F\right) C \\
& +\left(-G \eta p_{1}+B F-G \xi p \eta\right) D-B \delta \varepsilon-G\left(\delta p_{1}+\varepsilon p_{2}\right) \eta-F \delta \varepsilon, \\
b_{1}= & \left(\left((B+D) C+B D-G\left(p_{1}+p_{2}\right) \eta-\delta \varepsilon\right) A+\left((B+F) D-G \eta p_{2}+B F\right) C+(B F-\right. \\
& \left.\left.G \eta p_{1}\right) D-B \delta \varepsilon-G\left(\delta p_{1}+\varepsilon p_{2}\right) \eta-F \delta \varepsilon\right) E+\left(\left(-G \eta p_{2}+B D-G \xi p \eta\right) C-\right. \\
& \left.\left(G \eta p_{1}+G \xi p \eta\right) D-B \delta \varepsilon-G\left(\delta p_{1}+\varepsilon p_{2}\right) \eta\right) A+(-C D+\delta \varepsilon)(G \xi p \eta-B F), \\
b_{0}= & \left.\left(\left(-G \eta p_{2}+B D\right) C-D G \eta p_{1}-B \delta \varepsilon-G\left(\delta p_{1}+\varepsilon p_{2}\right) \eta\right) A-F B(-C D+\delta \varepsilon)\right) E+ \\
& A G \xi p \eta(-C D+\delta \varepsilon) .
\end{aligned}
$$


By the Routh-Hurwitz criterion, since $\lambda_{1}=-\mu$, the other eigenvalues are negative or have negative real parts if the following inequalities are satisfied:

$$
\begin{gathered}
b_{i}>0, \forall i=0,1,2,3,4, \quad b_{3} b_{4}>b_{2}, \quad b_{2} b_{3} b_{4}+b_{0} b_{4}>b_{2}^{2}+b_{1} b_{4}^{2}, \\
\left(b_{1} b_{4}-b_{0}\right)\left(b_{2} b_{3} b_{4}+b_{0} b_{4}-b_{2}^{2}-b_{1} b_{4}^{2}\right)>b_{0}\left(b_{3} b_{4}-b_{2}\right)^{2} .
\end{gathered}
$$

Under these conditions, the endemic equilibrium point is locally asymptotically stable. From the condition $b_{0}>0$ we obtain that $\mathscr{R}_{0}>1$. We get the following result:

Theorem 3.4 (Local stability of the endemic equilibrium point). The endemic equilibrium point $E_{1}$ of the system 3.1 exists if $\mathscr{R}_{0}>1$ and it is locally asymptotically stable only if $b_{i}>0, \forall i=0, \cdots, 4,\left(\mathscr{R}_{0}>\right.$ $1), b_{3} b_{4}>b_{2}, b_{2} b_{3} b_{4}+b_{0} b_{4}>b_{2}^{2}+b_{1} b_{4}^{2}$ and $\left(b_{1} b_{4}-b_{0}\right)\left(b_{2} b_{3} b_{4}+b_{0} b_{4}-b_{2}^{2}-b_{1} b_{4}^{2}\right)>b_{0}\left(b_{3} b_{4}-b_{2}\right)^{2}$.

\section{Simulations.}

4.1. SLIAR model. The ordinary differential equation solutions for the model were simulated using the initial conditions vector $\left(S_{0}, L_{0}, I_{0}, A_{0}, R_{0}\right)=(1988,0,12,0,0)$, and following parameters:

\begin{tabular}{|cllc|}
\hline Parameter & Meaning & Value & Reference \\
\hline$\beta$ & Transmission rate & 0.001 (person $\times$ day) & Assumed \\
\hline$\delta$ & Attenuation rate for asymptomatics & 0.5 non-dimensional & {$[4,18]$} \\
\hline$\kappa$ & Transition rate from latent to infected infectious & 0.526 day $^{-1}$ & {$[4,18]$} \\
\hline$\frac{1}{\kappa}$ & Average duration of incubation & 1.901 days & Estimated \\
\hline$p$ & $\begin{array}{l}\text { Probability of passing from latent to symptomatic } \\
\text { infected }\end{array}$ & 0.667 non-dimensional & {$[4,18]$} \\
\hline$\eta$ & Recovery rate for asymptomatic & 0.244 day ${ }^{-1}$ & {$[4,18]$} \\
\hline$\frac{1}{\eta}$ & $\begin{array}{l}\text { Average duration of infection, time in which an } \\
\text { asymptomatic infected person is contagious }\end{array}$ & 4.098 days & Estimated \\
\hline$f$ & Fraction of recovered symptomatic & 0.96 non-dimensional & Assumed \\
\hline$\alpha$ & Removal rate for symptomatics & 0.244 day ${ }^{-1}$ & {$[4,18]$} \\
\hline$\frac{1}{\alpha}$ & $\begin{array}{l}\text { Average duration of infection, time in which an } \\
\text { symptomatic infected person is contagious }\end{array}$ & 4.098 days $^{-1}$ & Estimated \\
\hline
\end{tabular}

Table 4.1: SLIAR model parameter values

Where the value of the recovery rate $f$ was calculated from the COVID-19 fatality rate reported for Peru [21] of $4 \%$, that is, $(1-f)=0.04$. Considering these parameters we can obtain a basic reproductive number as follows:

$$
\mathscr{R}_{0}=S_{0} \beta\left(\frac{\delta(1-p)}{\eta}+\frac{p}{\alpha}\right)=(1988)(0.001)\left(\frac{0.5(1-0.667)}{0.244}+\frac{0.667}{0.244}\right)=6.791 .
$$

According to the computer simulations we obtain the figure 4.1a and it can be deduced that the maximum number of symptomatic infected is 492 individuals on approximately day 12. Also, the maximum number of latent and asymptomatic infected people are about 500 and 245 individuals on day 9 and 11, respectively.

From the phase plane presented (Figure 4.1b), it shows that as the number of susceptible people decreases, the infected reach a maximum and then descend. This is because at the beginning, there was enough population to spread the epidemic, but once they reached a maximum number of infected, they begin to decline since there is no longer enough susceptible population to infect, giving way to the end of the outbreak. 


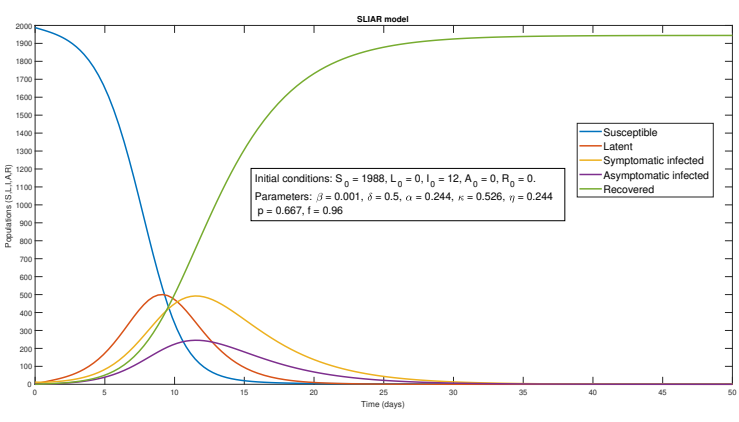

(a) Solution curves

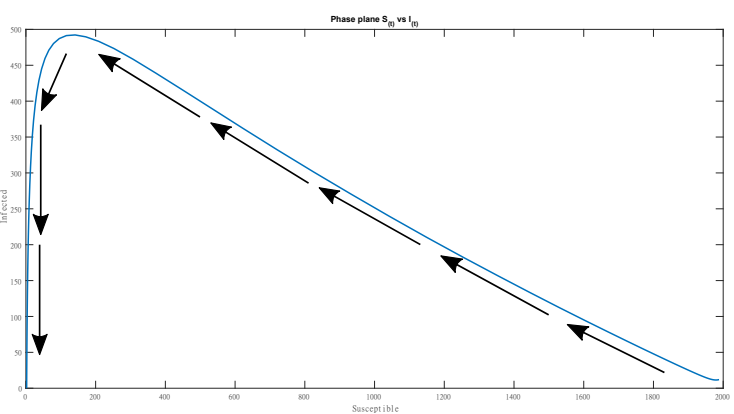

(b) Phase plane S-I

Figure 4.1: Solution curves and phase plane for disease-free case with $\mathscr{R}_{0}=6.791$

For the simulation sensitivity, one parameter was varied by some magnitude while the others were not, and the most sensitive parameters were selected (i.e., when varying a parameter by a small magnitude, it causes a significant change in the dynamics of the model solutions). For this, the MATLAB R2017a software was used, and different parameter values were computed, focusing on the mild infection curve as the main epidemiological aspect to consider, due to the large number of people in this epidemiological class, the figure 4.2 was obtained.

Regarding the transmission rate " $\beta$ " (Figure 4.2a), we observe that it is extremely sensitive since variations in the order of $10^{-3}$ cause the peak of the infected curve to shift. In this case, at a higher transmission rate, we reach the peak of infection faster and it is more pronounced. In the case of the removal rate of symptomatic individuals " $\alpha$ " (Figure 4.2b), we note that as it decreases, the epidemic outbreak will take place with greater speed and intensity.

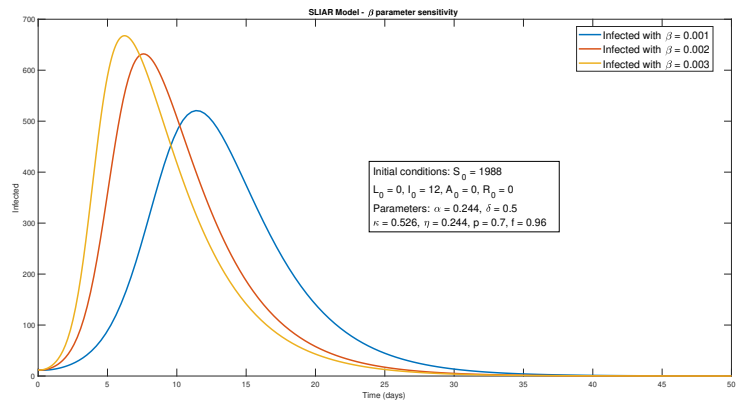

(a) Infected curve variation with different $\beta$ values

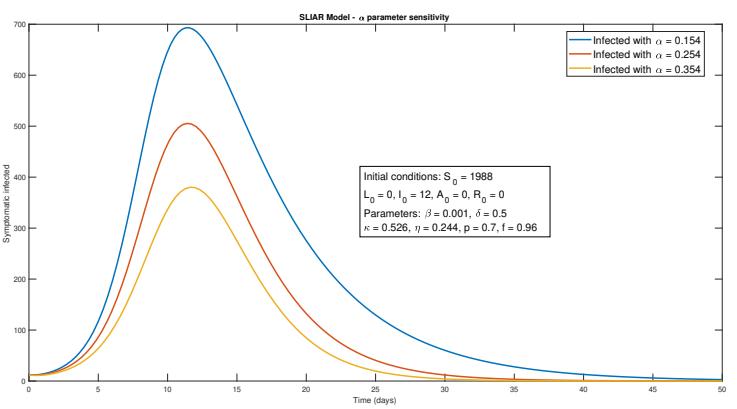

(b) Infected curve variation with different $\alpha$ values

Figure 4.2: Sensitivity results for $\beta$ and $\alpha$

4.2. SLIIAR model. The initial conditions used to simulate the long term behavior of the solutions are contained in the following vector $\left(S_{0}, L_{0}, I_{1_{0}}, I_{2_{0}}, A_{0}, R_{0}\right)=(1988,0,12,0,0,0)$.

In order to calculate $\beta$, we considered $S_{0} \beta=\bar{\beta}$, it means the number of susceptible individuals that one infectious person can contact to transmit the disease per time unit. For instance, from Li et al. [16] we can use the rank parameter values in the interval $\bar{\beta} \in[1.06,1.19]$ days $^{-1}$ at the beginning of the outbreak, considering in our case $S_{0}=1988$, so the rank of $\beta$ is $[5.33,5.99] \times 10^{-4}$ (person $\times$ day) $)^{-1}$.

The recruitment rate rank was assumed, taking into consideration a small population size according to our initial population (2000 people). The vaccination rate " $\kappa$ ", measures the vaccination speed, that is, the number of vaccinated people in proportion to the total size of the population per day. For example, if the health system can vaccinate 3000 people per day in a population of 100000 , we get a 0.03 vaccination rate, it means that $3 \%$ per day of susceptible population is vaccinated. We assume a uniform distribution in the interval $[0.4,0.6]$ to perform the sensitivity analysis for the attenuation rate for asymptomatics " $\xi$ ".

The complete list of parameters is the next: 


\begin{tabular}{|c|c|c|c|}
\hline Parameter & Meaning & Value & Reference \\
\hline$\Lambda$ & Recruitment rate & {$[1,20]$ person $\times$ day $^{-1}$} & Assumed $^{a}$ \\
\hline$\beta$ & Transmission rate & {$[5.33,9.99] \times 10^{-4}(\text { person } \times \text { day })^{-1}$} & Assumed $^{b}$ \\
\hline$\xi$ & Attenuation rate for asymptomatics & 0.51 non-dimensional & {$[1]$} \\
\hline$\eta$ & $\begin{array}{l}\text { Transition rate from latent to infected } \\
\text { infectious }\end{array}$ & {$[0.1724,0.2222] \mathrm{day}^{-1}$} & Estimated \\
\hline$\frac{1}{\eta}$ & Average duration of incubation & {$[4.5,5.8]$ days } & {$[15,20]^{c}$} \\
\hline$p_{1}$ & $\begin{array}{l}\text { Probability of passing from latent to } \\
\text { mild symptomatic }\end{array}$ & {$[0.10,0.83]$ non-dimensional } & Estimated $^{d}$ \\
\hline$p_{2}$ & $\begin{array}{l}\text { Probability of passing from latent to } \\
\text { severe symptomatic }\end{array}$ & {$[0,0.2]$ non-dimensional } & {$[1]$} \\
\hline $1-p_{1}-p_{2}$ & $\begin{array}{l}\text { Probability of passing from latent to } \\
\text { asymptomatic }\end{array}$ & {$[0.17,0.9]$ non-dimensional } & {$[1,2]$} \\
\hline$\gamma$ & Recovery rate & {$[0.0270,0.3125] \mathrm{day}^{-1}$} & Estimated \\
\hline$\frac{1}{\gamma}$ & $\begin{array}{l}\text { Average duration of infection, time } \\
\text { in which an infected person is con- } \\
\text { tagious }\end{array}$ & {$[3.2,37]$ days } & $\begin{array}{l}{[1,15,2,} \\
30]\end{array}$ \\
\hline$\omega_{1}$ & Lethality rate for mild symptomatics & {$[0.0032,0.01] \mathrm{day}^{-1}$} & {$[2,28]^{e}$} \\
\hline$\omega_{2}$ & $\begin{array}{l}\text { Lethality rate for severe symptomat- } \\
\text { ics }\end{array}$ & {$[0.000094,0.18] \mathrm{day}^{-1}$} & [1] \\
\hline$\mu$ & Per capita natural death rate & {$[3.1,5.144] \times 10^{-5} \mathrm{day}^{-1}$} & Assumed $^{f}$ \\
\hline$\delta$ & $\begin{array}{l}\text { Transition rate from mild to severe } \\
\text { symptomatic }\end{array}$ & {$[0.2,2.0] \mathrm{day}^{-1}$} & Assumed \\
\hline$\varepsilon$ & $\begin{array}{l}\text { Transition rate from severe to mild } \\
\text { symptomatic }\end{array}$ & {$[0.10,0.33] \mathrm{day}^{-1}$} & Assumed \\
\hline$\kappa$ & Vaccination rate & {$[0.001,0.1] \mathrm{day}^{-1}$} & Assumed $^{g}$ \\
\hline $\mathscr{R}_{0}$ & Basic reproduction number & {$[1.5,6.49]$ non-dimensional } & [17] \\
\hline \multicolumn{4}{|c|}{$\begin{array}{l}\left.{ }^{a} \text { Based on } 10 \text { person } \times \text { day }^{-1} \text { (Considering } S_{0}=367804\right) \text { [28], and } 271 \text { person } \times \text { day }^{-1} \text { (Considering } \\
\left.S_{0}=8998505\right)[27] .{ }^{b} \text { Other reported values are } 7.38 \times 10^{-7} \text { (person } \times \text { day) }{ }^{-1}[28],[0.62,3.11] \times 10^{-8} \\
\text { (person } \times \text { day) }{ }^{-1} \text { [27], }\{4.085,4.811,6.184,7.059\} \text { days }{ }^{-1} \text { [12] }{ }^{c} \text { Even though we considered this } \\
\text { rank, other values have been reported like } 6.4 \text { days [14], [4.1,7] days [1], } 7 \text { days [27, 24],2 days (for } \\
\text { average latency period) [1]. }{ }^{d} \text { Considering } p_{1} \text { as a complementary probability to } p_{2} \text { and }\left(1-p_{1}-p_{2}\right) \text {. } \\
{ }^{e} \text { Based on the disease-induced death rate. }{ }^{f} \text { Based on: } 2.74 \times 10^{-5} \text { days }^{-1} \text { [28], } 3.01 \times 10^{-5} \text { days }^{-1} \\
\text { [27]. }{ }^{g} \text { Based on the intervals }[0.001 ; 0.05] \\
{[26,23] .}\end{array}$} \\
\hline
\end{tabular}

Table 4.2: SLIIAR model parameter values for COVID-19

In the figure 4.3 , the solution curves in the disease-free equilibrium case $\left(\mathscr{R}_{0}=0.6699\right)$ show that the outbreak peaks in about 25 days and tend to be controlled in about two months (Figure 4.3b). Moreover, the phase planes (Figures 4.4a, 4.4b) indicate that the infected population tends to disappear and there is a remaining susceptible population. Furthermore, in the endemic case $\left(\mathscr{R}_{0}=2.0097\right)$, infected people reach a peak around the same time as in the previous case (Figure 4.5), but this peak is higher (Figure 4.5b), and the phase planes demonstrate the persistence of infected populations, whose trajectories converge to the endemic equilibrium point (red point). In both cases (Figures 4.4a, 4.6a), the phase planes describe that while the susceptible population is decreasing, the latent curve grows; after that, both populations reach a maximum and decrease immediately, but at the end of the dynamic, a growth in susceptible curve takes place by the recruitment effect. 


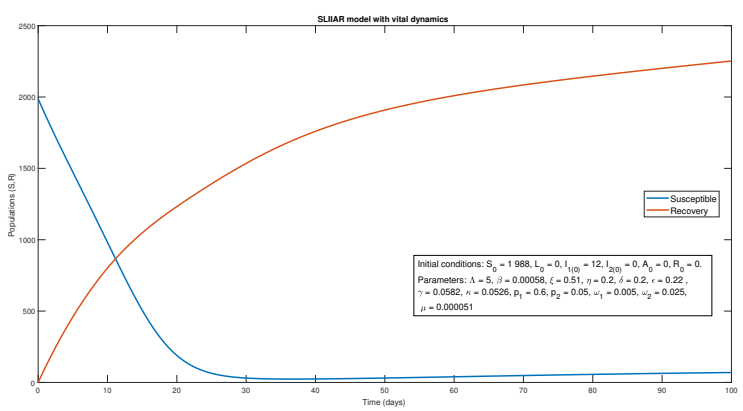

(a) Solutions curves for $S(t)$ and $R(t)$

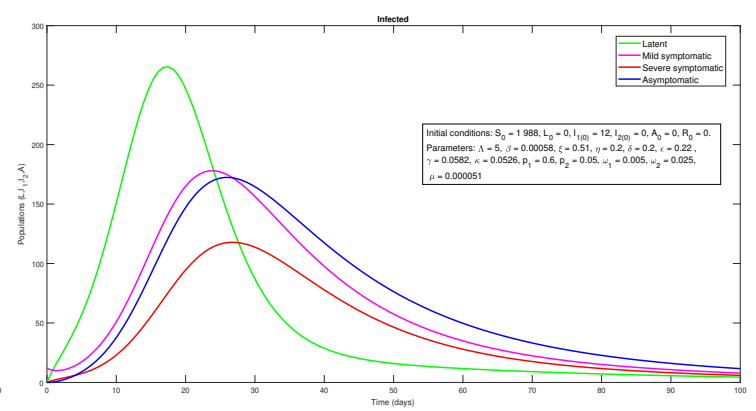

(b) Solutions curves for infected populations

Figure 4.3: Solution curves for disease-free case with $\mathscr{R}_{0}=0.6699$.

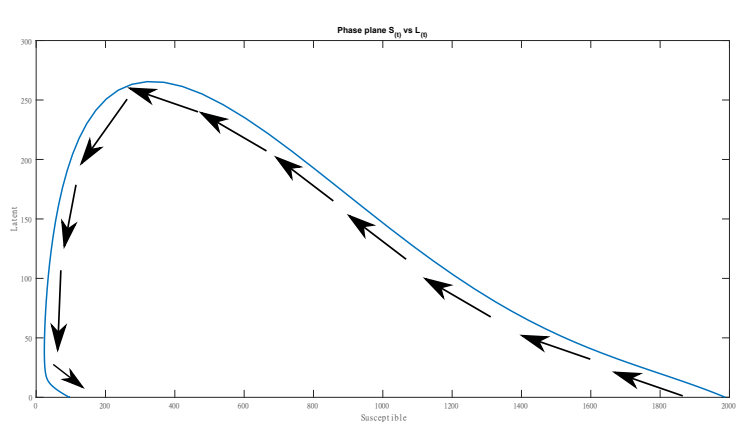

(a) Phase plane $S-L$

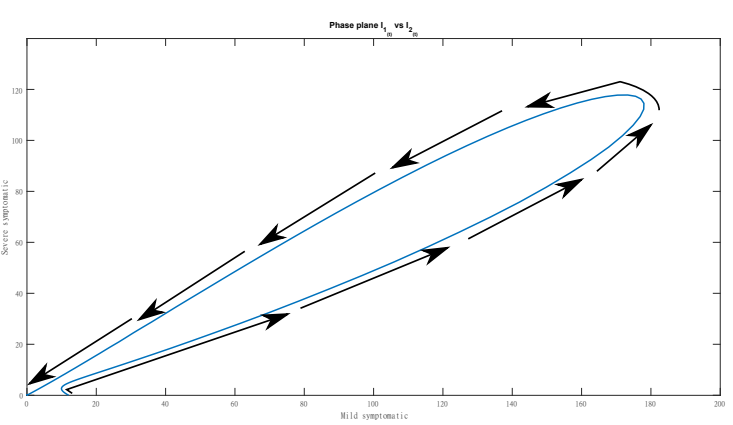

(b) Phase plane $I_{1}-I_{2}$

Figure 4.4: Phase plane curves for disease-free case with $\mathscr{R}_{0}=0.6699$.

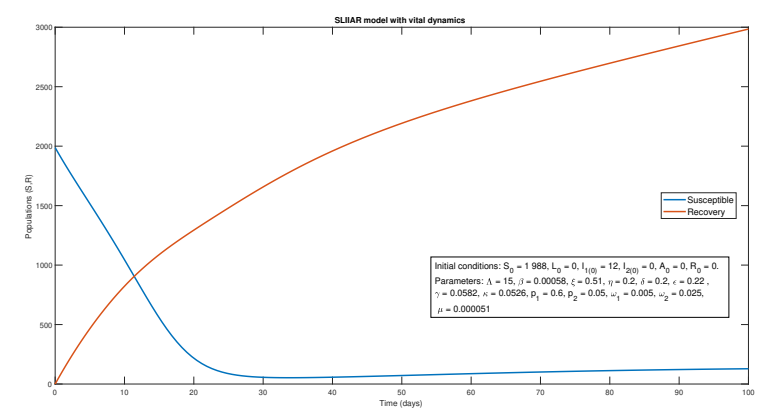

(a) Solutions curves for $S(t)$ and $R(t)$

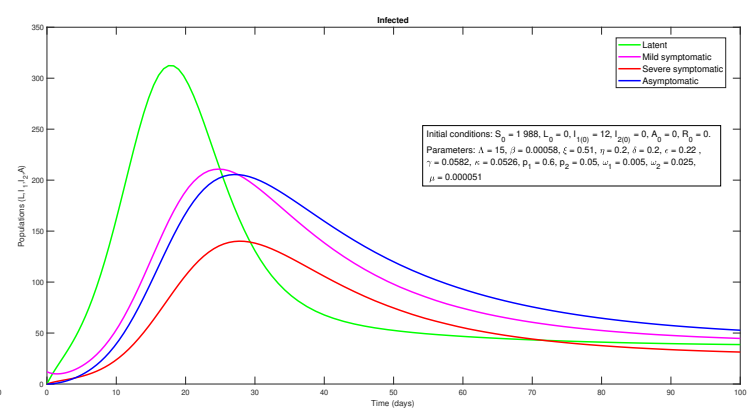

(b) Solutions curves for infected populations

Figure 4.5: Solution curves for endemic case with $\mathscr{R}_{0}=2.0097$. 


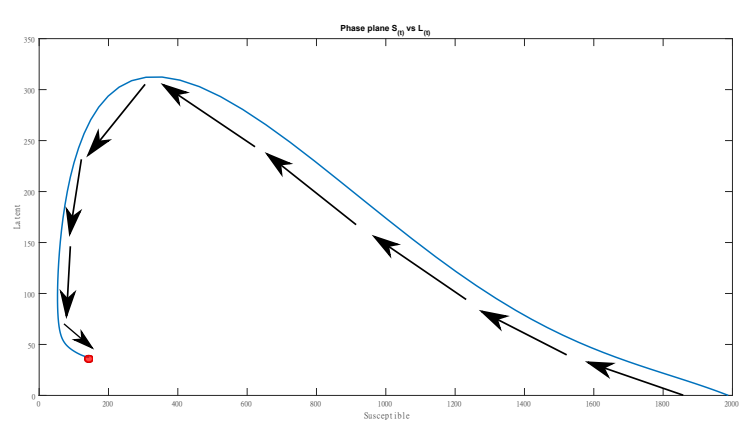

(a) Phase plane $S-L$

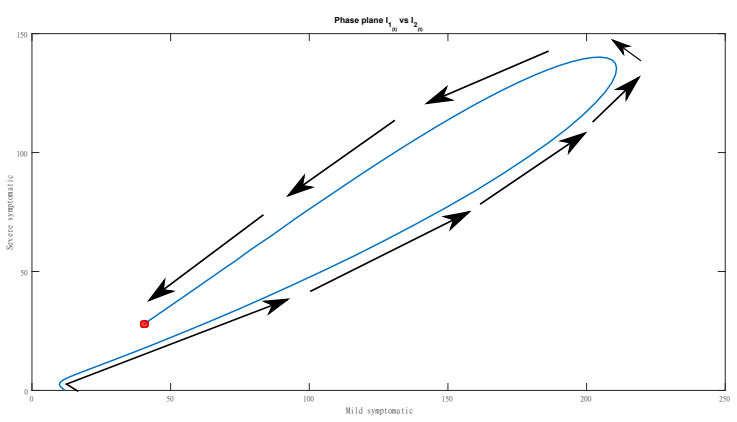

(b) Phase plane $I_{1}-I_{2}$

Figure 4.6: Phase plane curves for endemic case with $\mathscr{R}_{0}=2.0097$.

The simulation sensitivity was achieved for each parameter respect to the mild symptomatic population in the endemic case $\left(\mathscr{R}_{0}=2.0097\right)$. We identified that the solution curve is highly sensitive to the transmission rate $\beta$ (caused by variations in the order $10^{-4}$, Figure $4.7 \mathrm{~b}$ ), and less sensitive to the recruitment rate $\Lambda$ (Figure $4.7 \mathrm{a}$ ). In these cases, if we increase the parameter values, the mild symptomatic population reaches a higher peak in less time. Nevertheless, increasing the vaccination rate $\kappa$ (Figure $4.8 \mathrm{a}$ ) and the recovery rate $\gamma$ (Figure $4.8 \mathrm{~b}$ ) produces a smaller peak in less time.

Taking figure 4.7 into consideration, we observe that decreasing the transmission rate or the recruitment rate, causes a decrease in endemic cases in the long term. Due to this, controlling the spread by reducing the number of encounters between people (decreasing $\beta$ ) and the entry of new individuals into the system, for example, with focused quarantines (low $\Lambda$ values), can help reduce the number of patients to be treated and the overload of health systems. From figure 4.8 , it is possible to observe that increasing the daily vaccination rate (increasing $\kappa$ ) as well as decreasing the infectivity time of the disease (increasing the $\gamma$ value) with pharmacological treatments or biological agents, produce a lower number of patients.

According to the figure 4.9 , the vaccination rate " $\kappa$ " has the highest absolute PRCC index (0.87567), and this parameter also has an inverse proportional relationship (negative value) with respect to $\mathscr{R}_{0}$. It means that an increment in the vaccination rate results in a reduction in the $\mathscr{R}_{0}$ value, so the epidemic can decrease its severity. Furthermore, we discovered that $\mathscr{R}_{0}$ is highly sensitive and positively correlated to the recruitment rate " $\Lambda$ " (PRCC index: 0.84617), implying that an increase in the input flux will have a greater effect on $\mathscr{R}_{0}$ and the severity of an outbreak.The " $\gamma$ " recovery rate is also highly (PRCC index absolute value: 0.75271 ) and negatively correlated with $\mathscr{R}_{0}$, implying that decreasing the average infection time (i.e. increasing the recovery rate) will reduce both $\mathscr{R}_{0}$ and the spread of the outbreak.

Parameters such as the transmission rate $(\beta)$, the probability to become mild and severe infected $\left(p_{1}\right.$, $p_{2}$ ), and the attenuation rate for asymptomatic $(\xi)$ are positively correlated, all of them with PRCC values below 0.5 ; while parameters like the mortality rate for severe symptomatic $\left(\omega_{2}\right)$, the transition rate from mild to severe symptomatic $(\delta)$ and the natural death rate $(\mu)$ have an inverse proportional relationship with $\mathscr{R}_{0}$ (with PRCC absolute values less than 0.5 ).

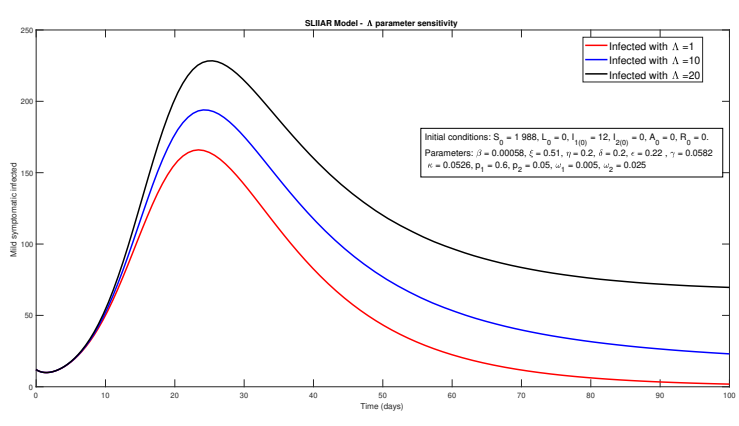

(a) $I_{1}$ curve variation with different $\Lambda$ values

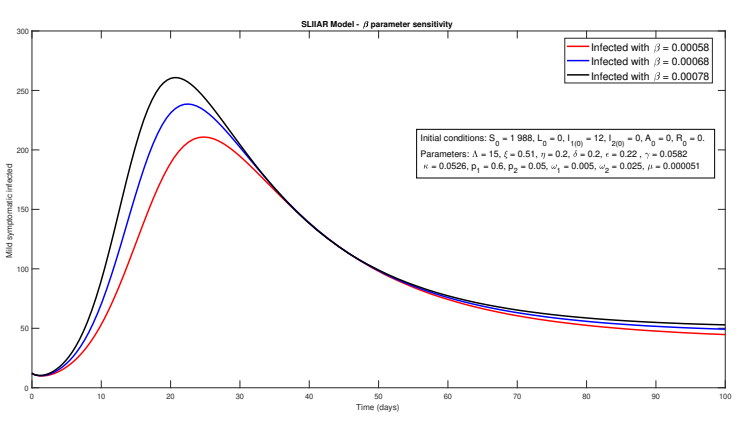

(b) $I_{1}$ curve variation with different $\beta$ values

Figure 4.7: Sensitivity results for $\Lambda$ and $\beta$ in the endemic case 


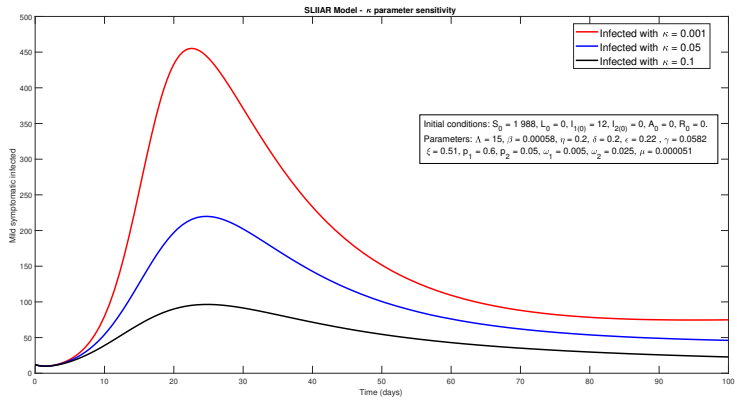

(a) $I_{1}$ curve variation with different $\kappa$ values

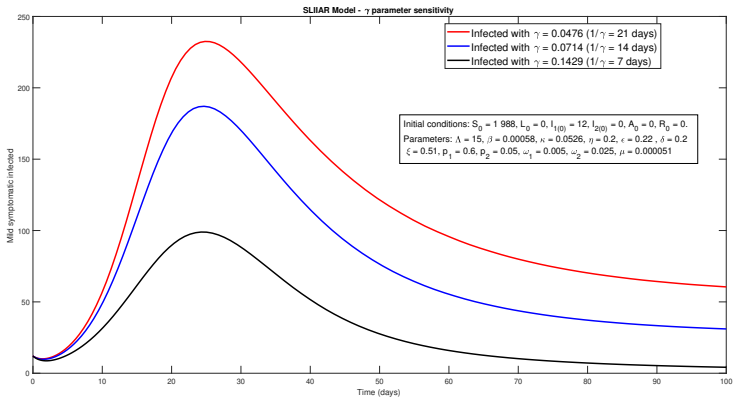

(b) $I_{1}$ curve variation with different $\gamma$ values

Figure 4.8: Sensitivity results for $\kappa$ and $\gamma$ in the endemic case

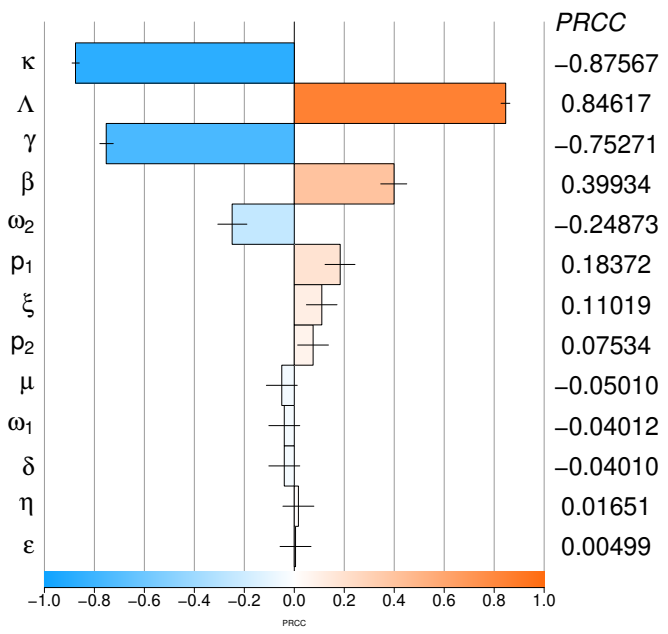

Figure 4.9: Sensitivity results for $\mathscr{R}_{0}$

5. Conclusions. The SLIIAR model with vital dynamics, vaccination, and symptomatological treatment was proposed. The disease-free equilibrium point exists and is locally asymptotically stable if $\mathscr{R}_{0}<1$, whereas the endemic equilibrium point exists and is locally asymptotically stable if $\mathscr{R}_{0}>1$. The simulations show that transmission and recruitment rates are important targets for outbreak control because they reduce the $\mathscr{R}_{0}$ value. Furthermore, if the vaccination speed is increased, a vaccination scheme with total immunity can reduce $\mathscr{R}_{0}$. Taking some public health measures like social distancing, quarantines, or a fast vaccination strategy, an outbreak can be efficiently controlled. The treatment does not appear to be very significant in the model dynamics, but the ulterior development of an efficient and rapid treatment effect can change this scenario. A global stability analysis can be developed and some hypotheses like partial immunity or reinfections can be considered in future work.

ORCID and License

Pedro Isaac Pesantes-Grados https : / / orcid.org/ $0000-0003-2370-1419$

Roxana López-Cruz https : / / orcid.org/0000-0002-7703-5784

This work is licensed under the Creative Commons - Attribution 4.0 International (CC BY 4.0)

\section{References}

[1] Abrams S, Wambua J, Santermans E, Willem L, Kuylen E, Coletti P, et al. Modelling the early phase of the Belgian COVID-19 epidemic using a stochastic compartmental model and studying its implied future trajectories. Epidemics. 2021;35(100449):100449.

[2] Aguilar JB, Faust JS, Westafer LM, Gutierrez JB, Investigating the impact of asymptomatic carriers on COVID-19 transmission,medRxiv (preprint) (2020).

[3] Amaya-Muvdi DA, Acercamientos a la epidemología de una enfermedad a través de sistemas compartimentales,B.S. thesis, Bogotá-Universidad de Los Andes, 2015. 
[4] Arino J, Brauer F, van den Driessche P, Watmough J, Wu J. Simple models for containment of a pandemic. J R Soc Interface. 2006;3(8):453-7.

[5] Arino J, Brauer F, van den Driessche P, Watmough J, Wu J. A final size relation for epidemic models. Math Biosci Eng. 2007;4(2):159-75.

[6] Arino J, Portet S. A simple model for COVID-19. Infect Dis Model. 2020;5:309-15.

[7] Arino J. Mathematical epidemiology in a data-rich world. Infect Dis Model. 2020;5:161-88.

[8] Brauer F. Some simple epidemic models. Math Biosci Eng. 2006;3(1):1-15.

[9] Castillo-Salgado C, Mujica Ó J, Loyola E, Canela J , Módulos de principios de epidemiología para el control de enfermedades, segunda edición. Organización Panamericana de la Salud. Washington D.C., 2011.

[10] Chen J. Pathogenicity and transmissibility of 2019-nCoV-A quick overview and comparison with other emerging viruses. Microbes Infect. 2020;22(2):69-71.

[11] Diekmann O, Heesterbeek JAP, Roberts MG. The construction of next-generation matrices for compartmental epidemic models. J R Soc Interface. 2010;7(47):873-85.

[12] Kim S, Seo YB, Jung E. Prediction of COVID-19 transmission dynamics using a mathematical model considering behavior changes in Korea. Epidemiol Health. 2020;42:e2020026.

[13] Kermack WO, McKendrick AG. A contribution to the mathematical theory of epidemics. Proc R Soc Lond A Math Phys Sci. 1927;115(772):700-21.

[14] Lai C-C, Shih T-P, Ko W-C, Tang H-J, Hsueh P-R. Severe acute respiratory syndrome coronavirus 2 (SARS-CoV-2) and coronavirus disease-2019 (COVID-19): The epidemic and the challenges. Int J Antimicrob Agents. 2020;55(3):105924.

[15] Lauer SA, Grantz KH, Bi Q, Jones FK, Zheng Q, Meredith HR, et al. The incubation period of Coronavirus disease 2019 (COVID-19) from publicly reported confirmed cases: Estimation and application. Ann Intern Med. 2020;172(9):577-82.

[16] Li R, Pei S, Chen B, Song Y, Zhang T, Yang W, et al. Substantial undocumented infection facilitates the rapid dissemination of novel coronavirus (SARS-CoV-2). Science. 2020;368(6490):489-93.

[17] Liu Y, Gayle AA, Wilder-Smith A, Rocklöv J. The reproductive number of COVID-19 is higher compared to SARS coronavirus. J Travel Med. 2020;27(2). Available from: http://dx.doi.org/10.1093/jtm/taaa021.

[18] Longini IM Jr, Halloran ME, Nizam A, Yang Y. Containing pandemic influenza with antiviral agents. Am J Epidemiol. 2004;159(7):623-33.

[19] Marino S, Hogue IB, Ray CJ, Kirschner DE. A methodology for performing global uncertainty and sensitivity analysis in systems biology. J Theor Biol. 2008;254(1):178-96.

[20] MINSA, Resolución Ministerial-139-2020-MINSA: Documento Técnico - Prevención y atención de personas afectadas por COVID-19 en el Perú,(MINSA) (2020) 6-9.

[21] MINSA, Sala situacional COVID-19 Perú, updated to 28/09/2020 [Reviewed 29/09/2020], Ministerio de Salud de la República del Perú (MINSA). Available from: https://covid19.minsa.gob.pe/sala_situacional.asp.

[22] Rasmussen AL, Popescu SV. SARS-CoV-2 transmission without symptoms. Science. 2021;371(6535):1206-7.

[23] Romero-Brufau S, Chopra A, Ryu AJ, Gel E, Raskar R, Kremers W, et al. Public health impact of delaying second dose of BNT162b2 or mRNA-1273 covid-19 vaccine: simulation agent based modeling study. BMJ. 2021;373:n1087.

[24] Tang B, Wang X, Li Q, Bragazzi NL, Tang S, Xiao Y, et al. Estimation of the transmission risk of the 2019-nCoV and its implication for public health interventions. J Clin Med. 2020;9(2):462.

[25] van den Driessche P, Watmough J. Reproduction numbers and sub-threshold endemic equilibria for compartmental models of disease transmission. Math Biosci. 2002;180(1-2):29-48.

[26] Wintachai P, Prathom K. Stability analysis of SEIR model related to efficiency of vaccines for COVID-19 situation. Heliyon. 2021;7(4):e06812.

[27] Yang CY, Wang J. A mathematical model for the novel coronavirus epidemic in Wuhan, China. Math Biosci Eng. 2020;17(3):2708-24.

[28] Yang CY, Wang J. Modeling the transmission of COVID-19 in the US-A case study. Infectious Disease Modelling 2021; 6: 195-211.

[29] Zhang T, Liu J, Teng Z. Existence of positive periodic solutions of an SEIR model with periodic coefficients. Appl Math (Prague). 2012;57(6):601-16.

[30] Zhou F, Yu T, Du R, Fan G, Liu Y, Liu Z, et al. Clinical course and risk factors for mortality of adult inpatients with COVID-19 in Wuhan, China: a retrospective cohort study. Lancet. 2020;395(10229):1054-62. 